\title{
The interstellar medium in Andromeda's dwarf spheroidal galaxies - II. Multiphase gas content and ISM conditions
}

\author{
Ilse De Looze, ${ }^{1,2,3 \star}$ Maarten Baes, ${ }^{2}$ Diane Cormier, ${ }^{4}$ Hiroyuki Kaneko, ${ }^{5}$ \\ Nario Kuno, ${ }^{6}$ Lisa Young, ${ }^{7,8}$ George J. Bendo, ${ }^{9}$ Médéric Boquien, $, 3,10$ \\ Jacopo Fritz, ${ }^{2,11}$ Gianfranco Gentile, ${ }^{2}$ Robert C. Kennicutt, ${ }^{3}$ Suzanne C. Madden, ${ }^{12}$ \\ Matthew W. L. Smith ${ }^{13}$ and Christine D. Wilson ${ }^{14}$ \\ ${ }^{1}$ Department of Physics and Astronomy, University College London, Gower Street, London WC1E 6BT, UK \\ ${ }^{2}$ Sterrenkundig Observatorium, Universiteit Gent, Krijgslaan 281 S9, B-9000 Gent, Belgium \\ ${ }^{3}$ Institute of Astronomy, University of Cambridge, Madingley Road, Cambridge CB3 OHA, UK \\ ${ }^{4}$ Zentrum für Astronomie der Universität Heidelberg, ITA, Albert-Ueberle Str. 2, D-69120 Heidelberg, Germany \\ ${ }^{5}$ Nobeyama Radio Observatory, Minamimaki, Minamisaku, Nagano 384-1305, Japan \\ ${ }^{6}$ Faculty of Pure and Applied Sciences, University of Tsukuba, 1-1-1 Tennoudai, Tsukuba, Ibaraki 350-8577, Japan \\ ${ }^{7}$ Physics Department, New Mexico Institute of Mining and Technology, Socorro, NM 87801, USA \\ ${ }^{8}$ Academia Sinica Institute of Astronomy and Astrophysics, PO Box 23-141, Taipei 10617, Taiwan, Republic of China \\ ${ }^{9}$ UK ALMA Regional Centre Node, Jodrell Bank Centre for Astrophysics, School of Physics and Astronomy, University of Manchester, Oxford Road, \\ Manchester M13 9PL, UK \\ ${ }^{10}$ Unidad de Astronomía, Fac. Cs. Básicas, Universidad de Antofagasta, Avda. U. de Antofagasta 02800, Antofagasta, Chile \\ ${ }^{11}$ Centro de Radioastronomía y Astrofísica, CRyA, UNAM, Campus Morelia, A.P. 3-72, C.P. 58089 Michoacán, Mexico \\ ${ }^{12}$ Laboratoire AIM, CEA, Université Paris VII, IRFU/Service d'Astrophysique, Bat. 709, F-91191 Gif-sur-Yvette, France \\ ${ }^{13}$ School of Physics and Astronomy, Cardiff University, Queens Buildings, The Parade, Cardiff CF24 3A A, UK \\ ${ }^{14}$ Department of Physics and Astronomy, McMaster University, Hamilton, ON L8S 4M1, Canada
}

\begin{abstract}
We make an inventory of the interstellar medium material in three low-metallicity dwarf spheroidal galaxies of the Local Group (NGC 147, NGC 185 and NGC 205). Ancillary H I, $\mathrm{CO}$, Spitzer Infrared Spectrograph spectra, $\mathrm{H} \alpha$ and X-ray observations are combined to trace the atomic, cold and warm molecular, ionized and hot gas phases. We present new Nobeyama CO(1-0) observations and Herschel SPIRE FTS [C I] observations of NGC 205 to revise its molecular gas content. We derive total gas masses of $M_{\mathrm{g}}=1.9-5.5 \times 10^{5} \mathrm{M}_{\odot}$ for NGC 185 and $M_{\mathrm{g}}=8.6-25.0 \times 10^{5} \mathrm{M}_{\odot}$ for NGC 205. Non-detections combine to an upper limit on the gas mass of $M_{\mathrm{g}} \leq 0.3-2.2 \times 10^{5} \mathrm{M}_{\odot}$ for NGC 147. The observed gas reservoirs are significantly lower compared to the expected gas masses based on a simple closed-box model that accounts for the gas mass returned by planetary nebulae and supernovae. The gas-to-dust mass ratios GDR 37-107 and 48-139 are also considerably lower compared to the expected GDR $\sim 370$ and 520 for the low metal abundances in NGC $185\left(0.36 \mathrm{Z}_{\odot}\right)$ and NGC 205 $\left(0.25 \mathrm{Z}_{\odot}\right)$, respectively. To simultaneously account for the gas deficiency and low gas-todust ratios, we require an efficient removal of a large gas fraction and a longer dust survival time ( $\sim 1.6 \mathrm{Gyr})$. We believe that efficient galactic winds (combined with heating of gas to sufficiently high temperatures in order for it to escape from the galaxy) and/or environmental interactions with neighbouring galaxies are responsible for the gas removal from NGC 147, NGC 185 and NGC 205.
\end{abstract}

Key words: ISM: evolution-galaxies: dwarf-galaxies: individual: NGC 147, NGC 185, NGC 205 - Local Group-infrared: ISM. 


\section{INTRODUCTION}

Dwarf spheroidal galaxies (dSph) dominate the overall galaxy population in the Universe at the low mass end. With most dSphs residing in groups and clusters of galaxies, environmental effects are thought to play an important role in the formation and evolution of the dSph galaxy population. Studying the properties of the interstellar medium (ISM) in dSph galaxies, in combination with their star formation histories (SFH), can give us clues to their formation processes (e.g. Tolstoy, Hill \& Tosi 2009) and the role of environmental processes in their evolution (e.g. Boselli et al. 2008). Being the most prominent $\mathrm{dSph}$ residents of the Local Group, the three dwarf satellites of Andromeda, NGC 147, NGC 185 and NGC 205, offer the best opportunity to study the ISM of dSph galaxies in the nearby Universe.

In De Looze et al. (2016), we focused on the dust reservoirs in the three dSph satellites of Andromeda and show that the observed dust masses in NGC 185 and NGC 205 are significantly higher compared to the estimated metal enrichment from evolved stars and supernova remnants. Although uncertainties on the dust yields from asymptotic giant branch (AGB) and supernovae might affect the estimated dust production rates, the observed dust masses exceed predictions by an order of magnitude and can only be explained by efficient interstellar grain growth or longer dust survival times (3-6 Gyr).

Based on observational constraints of the evolved stellar populations, the dSph satellites NGC 147, NGC 185 and NGC 205 are shown to be characterized by significantly lower gas masses compared to the predicted material returned by evolved stars and the left-over gas reservoir that remains after previous star formation (SF) episodes (Sage, Welch \& Mitchell 1998; Welch, Sage \& Mitchell 1998; De Looze et al. 2012). The gas deficiency in NGC 205 was attributed to the environmental interactions with parent galaxy Andromeda and/or efficient stellar feedback (De Looze et al. 2012). To rule out that an important gaseous ISM reservoir has been overlooked in previous studies, we require an accurate quantification of the interstellar material (its mass and properties) in $\mathrm{dSphs}$ in combination with models that account for the gas mass returned by the evolved stellar population and supernovae. In this paper, we make a revised inventory and updated analysis of the gaseous reservoir in the three dSph satellite galaxies of Andromeda by taking into account all significant phases of their ISM. We present a new Nobeyama $\mathrm{CO}(1-0)$ map of the southern regions in NGC 205, Herschel PACS line spectroscopy observations for NGC 185 and Herschel SPIRE FTS spectroscopy observations for NGC 205. We furthermore use the ancillary data of other gaseous components tracing the atomic gas $(\mathrm{HI})$, cold $(\mathrm{CO}), \mathrm{CO}$-dark $\left(\left[\mathrm{C}_{\mathrm{I}}\right]\right)$ and warm molecular gas $\left(\mathrm{H}_{2}\right.$ rotational lines), ionized gas $(\mathrm{H} \alpha)$ and hot $\mathrm{X}$-ray emitting gas.

The description of the star formation histories (SPH) and characterization of the most recent star formation rates (SFRs) and metal abundances for the three galaxies under study have been outlined in the three paragraphs below. Table 1 presents an overview of the general properties and available observational constraints for each of the galaxies. In Section 2, we present the new Nobeyama Radio Observatory (NRO) 45 m CO(1-0) observations, Herschel PACS and SPIRE spectroscopy data and the ancillary data sets used to analyse the gaseous reservoirs in NGC 147, NGC 185 and NGC 205. To learn more about the physical gas conditions, we analyse the origin of the [C II] emission in NGC 185 (3.1), quantify the photoelectric efficiency (3.2) and compare the emission of gas tracers to photo-dissociation models (3.3). Several observations are
Table 1. Overview of the galaxy characteristics and availability of observational constraints for the three $\mathrm{dSph}$ galaxies NGC 147, NGC 185 and NGC 205. Total galaxy masses (including baryonic and dark matter) have been taken from De Rijcke et al. (2006). The SFRs are representative for the SF activity during the last $1 \mathrm{Gyr}$ (see Section 1.2). The metallicity is constrained by the average oxygen abundance of PNe (see Section 1.3). The symbols indicate that the galaxy has been observed and detected (' $x$ '), observed but not detected ('<') or was not observed ('o') for that specific gas tracer.

\begin{tabular}{|c|c|c|c|}
\hline Galaxy properties & NGC 147 & NGC 185 & NGC 205 \\
\hline Distance $(\mathrm{kpc})$ & 675 & 616 & 824 \\
\hline Galaxy mass $\left(10^{8} \mathrm{M}_{\odot}\right)$ & 3.0 & 2.6 & 10.2 \\
\hline $\operatorname{SFR}\left(\mathrm{M}_{\odot} \mathrm{yr}^{-1}\right)$ & $0^{a}$ & $6.6 \times 10^{-4}$ & $7.0 \times 10^{-}$ \\
\hline Metallicity $[12+\log \mathrm{O} / \mathrm{H}]$ & 8.06 & 8.25 & 8.08 \\
\hline Observational constraints & NGC 147 & NGC 185 & NGC 205 \\
\hline $\mathrm{HI}_{\mathrm{I}}$ & $<$ & $\mathrm{x}$ & $\mathrm{x}$ \\
\hline $\mathrm{CO}(1-0)$ & $<$ & $\mathrm{x}$ & $\mathrm{x}$ \\
\hline Warm $\mathrm{H}_{2}$ (Spitzer IRS) & o & $\mathrm{x}$ & o \\
\hline [C II], [O I] (Herschel PACS) & o & $\mathrm{x}$ & $\mathrm{x}$ \\
\hline [C I] (Herschel SPIRE FTS) & o & o & $<$ \\
\hline [N II] (Herschel SPIRE FTS) & o & o & $<$ \\
\hline $\mathrm{H} \alpha$ & o & $\mathrm{x}$ & $<$ \\
\hline X-ray & $<$ & $<$ & $<$ \\
\hline
\end{tabular}

Note. ${ }^{a}$ There is no evidence of any recent star formation activity in NGC 147 (Han et al. 1997).

combined to derive the total gas content in the three dSph galaxies (Section 4). Section 5 investigates the position of dSphs on the local Kennicutt-Schmidt relation. Combining dust and gas mass reservoirs, we derive gas-to-dust mass ratios for NGC 185 and NGC 205 in Section 6. The ISM mass budget in the three dSph companions of Andromeda (NGC 147, NGC 185, NGC 205) is compared to a simple closed box model and discussed in the light of galaxy evolution processes in Section 7. The main results are summarized in Section 8. Throughout this paper, we adopt distances of $675 \pm 27$, $616 \pm 26$ and $824 \pm 27 \mathrm{kpc}$ to NGC 147, NGC 185 and NGC 205 (McConnachie et al. 2005), respectively.

\subsection{Star formation history}

$\mathrm{dSph}$ galaxies are considered to form their stars in a limited number of SF episodes lasting a few Gyroyears and clearly separated by quiescent periods (e.g. Lanfranchi \& Matteucci 2004). Martins et al. (2012) determined that the SF in NGC 185 has taken place in three major episodes separated by quiescent periods without any significant SF activity. The first SF episode, during which most of the stellar content was produced, took place $\sim 10$ Gyr ago in NGC 185 (Geha et al. 2015), resulting in a stellar population with an iron abundance of $[\mathrm{Fe} / \mathrm{H}] \sim-1.0$. After the first SF episode, which lasted a few Gyroyears, NGC 185 had a long quiescent period without any significant SF activity. The presence of an intermediateage population (2-3 Gyr old) suggested a secondary SF episode. This second cycle of SF was considered to be the result of the build-up of mass-loss from evolved stars and/or planetary nebulae (PNe; Welch, Mitchell \& Yi 1996; Davidge 2005). A similar old and intermediate stellar population has been observed in NGC 147. The old stellar population in NGC 147 has a mean age ( $7.5 \mathrm{Gyr})$ and metallicity $([\mathrm{Fe} / \mathrm{H}] \sim-0.7)$, making the stars considerably younger in this galaxy and more metal-rich compared to the stars in NGC 185 (with mean age of $10 \mathrm{Gyr}$ and $[\mathrm{Fe} / \mathrm{H}] \sim-1.0$ ). This suggests that the bulk of stars in NGC 185 already formed at an earlier epoch 
(Geha et al. 2015). In the central regions of NGC 185, a more recent SF episode took place that started a few 100 Myr ago. NGC 147 shows no signs of any recent SF activity (Han et al. 1997). An old stellar population (10 Gyr; Bica, Alloin \& Schmidt 1990) also dominates the overall stellar content of NGC 205, while a plume of bright blue star clusters in the central region of this galaxy was already identified $60 \mathrm{yr}$ ago (Baade 1951; Hodge 1973).

\subsection{Star formation rates}

In NGC 147, no significant SF activity has taken place during the last 1 Gyr (Han et al. 1997). The SFR $\left(\sim 6.6 \times 10^{-4} \mathrm{M}_{\odot} \mathrm{yr}^{-1}\right)$ in the central regions of NGC 185 (inner 118 arcsec) over the last $\sim 1 \mathrm{Gyr}$ has been determined from colour-magnitude diagrams by MartínezDelgado, Aparicio \& Gallart (1999). The total SFR $\sim 82 \times 10^{-4}$ $\mathrm{M}_{\odot} \mathrm{yr}^{-1}$ (over the entire lifetime of the galaxy) in those central regions is significantly higher and consistent with a SPH where most of the stars have been formed in the first few Gyroyears after the formation of the galaxy (Martínez-Delgado et al. 1999). The latter central SFR should also be considered as an upper limit given that the inner regions are affected by crowding and every blue object has been assumed to be an individual star. In a similar way, the SFR $(\sim$ $7.0 \times 10^{-4} \mathrm{M}_{\odot} \mathrm{yr}^{-1}$ ) for the central $28 \operatorname{arcsec} \times 26 \operatorname{arcsec}$ region in NGC 205 has been derived from colour-magnitude diagrams for the stars produced between $\sim 62$ and $\sim 335$ Myr ago over a time period of $\sim 273 \mathrm{Myr}$ (Monaco et al. 2009). The latter SFR estimates derived from colour-magnitude diagrams should be more reliable compared to the standard SFR calibration that require sufficient sampling of stellar ages and assume a continuous SF activity.

\subsection{Metal abundance determination}

For NGC 147, the mean metallicity $12+\log (\mathrm{O} / \mathrm{H}) \sim 8.06$ (or $0.23 \mathrm{Z}_{\odot}$ ) is estimated from observations of eight $\mathrm{PNe}$ (Gonçalves et al. 2007). The latter metallicity based on $\mathrm{PNe}$ is not very different from the metallicity derived for the old stellar population $([\mathrm{Fe} / \mathrm{H}]$ $\sim-0.7$; Geha et al. 2015), which is in line with a low SF activity and negligible metal enrichment during the last few Gyroyears in NGC 147. The metallicity in NGC 185 is estimated by averaging the oxygen abundances derived for five central $\mathrm{PNe}$ reported by Richer \& McCall (2008), resulting in $12+\log (\mathrm{O} / \mathrm{H}) \sim 8.25$ or $Z \sim$ $0.36 \mathrm{Z}_{\odot}$ (assuming a solar oxygen abundance of $12+\log (\mathrm{O} / \mathrm{H}) \sim$ 8.69; Asplund et al. 2009). Similarly, Gonçalves et al. (2012) find a mean oxygen abundance of $12+\log (\mathrm{O} / \mathrm{H}) \sim 8.20$ or $Z \sim 0.32 \mathrm{Z}_{\odot}$ for NGC 185 based on the independent observations for four of the same PNe. In the same way, we derive the mean oxygen abundance $12+\log (\mathrm{O} / \mathrm{H}) \sim 8.08$ (or $0.25 \mathrm{Z}_{\odot}{ }^{1}$ based on $14 \mathrm{PNe}$ in NGC 205 (Gonçalves et al. 2014).

We caution that the abundances of PNe (probing the evolutionary products of the intermediate-mass stars) might be lower with respect to the abundances in $\mathrm{H}$ II regions (which probe the initial phases of massive stellar evolution) due to their different stages of evolution. Based on the comparison studies of elemental abundances derived for $\mathrm{H}_{\mathrm{II}}$ regions and PNe in NGC 300 (Stasińska et al. 2013) and M 33 (Bresolin et al. 2010; Magrini et al. 2010), we consider a maximum offset of 0.15 dex between the abundances from $\mathrm{H}_{\text {II }}$ regions and $\mathrm{PNe}$.

\footnotetext{
${ }^{1}$ The latter mean oxygen abundance is a bit higher compared to the mean value $(12+\log (\mathrm{O} / \mathrm{H}) \sim 7.80)$ used in De Looze et al. (2012) that was calculated as the average of 13 PNe analysed by Richer \& McCall (2008).
}

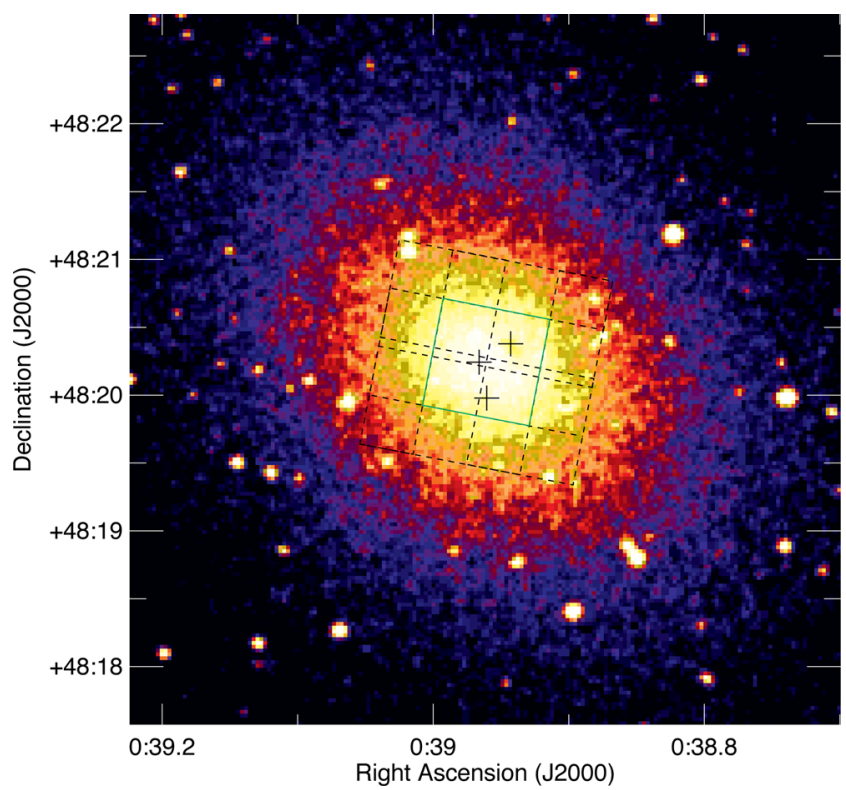

Figure 1. Optical DSS image of NGC 185 overlaid with the footprint of the Herschel PACS [C II] and [O I] observations as black dashed and blue solid lines, respectively. The Spitzer IRS north, central and south extraction positions from Marleau, Noriega-Crespo \& Misselt (2010) are indicated as black crosses.

\section{DATA}

\subsection{PACS spectroscopy data of NGC 185}

With the PACS spectrometer onboard Herschel (Pilbratt et al. 2010), we observed $3 \times 3$ raster maps of the fine-structure [C $\mathrm{II}] 158 \mu \mathrm{m}$ line in a chop-nod observing mode with two repetitions, which covers the central $100 \operatorname{arcsec} \times 100 \operatorname{arcsec}$ area in NGC 185. The [O I] $63 \mu \mathrm{m}$ line was observed only in one raster position covering the central $50 \operatorname{arcsec} \times 50$ arcsec with eight repetitions (see Fig. 1). The PACS spectra of [C II] (ObsID 1342247543) and [O I] (ObsID 1342247544) lines in the central regions of NGC 185 were taken on 2012 July 30 . The data cubes were processed from Level 0 using the telescope normalization pipeline in HIPE v12.0, with version 65 of the calibration files. After the spectral flat-fielding, the HIPE data cubes were exported to PACSman v.3.5.2 (Lebouteiller et al. 2012) to perform the line fitting and map projection. The line fitting was done on the full data cloud for each of the 25 spaxels. ${ }^{2}$ The line spectra in each spaxel were modelled with a second-order polynomial and Gaussian function to reproduce the continuum and line emission. The line fit parameters were optimized through a robust non-linear square curve fitting procedure. The spaxels were combined by drizzling to create final intensity maps with pixel size of 3.13 arcsec (i.e. about one-third of the size of a single spaxel). The full width at half-maximum (FWHM) of the PACS beam at 63 and $158 \mu \mathrm{m}$ corresponds to $\sim 9.5$ and $11.5 \operatorname{arcsec}$ (or 28 and $32 \mathrm{pc}$ at the distance of NGC 185), with a spectral resolution of $\sim 90$ and $\sim 240 \mathrm{~km} \mathrm{~s}^{-1}$, respectively (see PACS Observer's Manual). The uncertainties inherent to observational noise and line fitting were determined by PACSman. An additional noise factor accounting for a 15 per cent and 16 per cent calibration error (Poglitsch et al. 2010)

\footnotetext{
${ }^{2}$ Spaxels are spatial pixels that each contain a whole spectrum for a pixel on the sky. For the PACS spectrometer, spaxels have a size of $9.4 \operatorname{arcsec} \times$ 9.4 arcsec.
} 


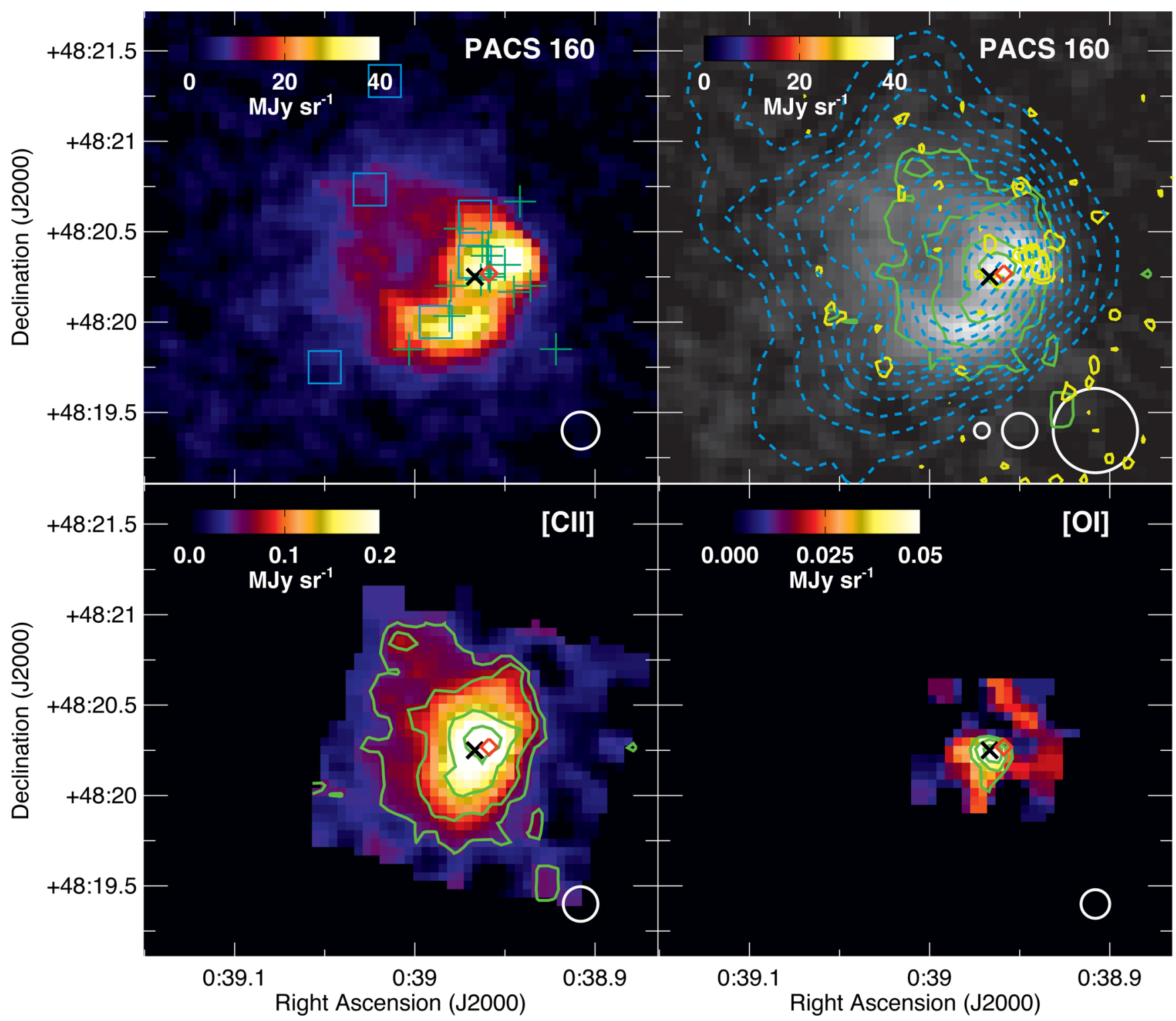

Figure 2. Top panels: Herschel PACS $160 \mu \mathrm{m}$ maps of NGC 185 with the positions of the blue stars (or star clusters) identified by Baade (1951) (green crosses) and clumps in the $\mathrm{H}_{\mathrm{I}}$ distribution (cyan squares) overlaid in the left-hand panel. In the right-hand panel, the contours of $\mathrm{H}_{\mathrm{I}}, \mathrm{CO}$ and [C $\mathrm{CI}$ observations are overlaid as blue dashed, yellow and green solid lines, respectively, at their original resolution. The beam sizes of the CO (FWHM $\sim 5$ arcsec), [C II] (FWHM $\sim 11.5 \mathrm{arcsec})$ and $\mathrm{H}$ I $(\mathrm{FWHM} \sim 28 \mathrm{arcsec})$ are indicated in the bottom right corner. The latter figures were taken from De Looze et al. (2016). Bottom panels: Herschel PACS [C II] (left) and [O I] (right) maps of NGC 185. Beamsizes are indicated in the lower right corner of the respective images. The green contours indicate the 3,5,10 and $15 \sigma \mathrm{S} / \mathrm{N}$ levels for $\left[\mathrm{C}_{\mathrm{II}}\right]$ and the 3,5 and $8 \sigma \mathrm{S} / \mathrm{N}$ levels for [O $\left.\mathrm{I}\right]$. The positions of supernova remnant SNR-1 and the centre of NGC 185 are indicated with a red diamond and black cross, respectively. All images have the same field of view (FOV).

at 63 and $158 \mu \mathrm{m}$ was added in quadrature to the former uncertainties. This calibration uncertainty accounts for the 11-12 percent absolute calibration uncertainty (at 63 and $158 \mu \mathrm{m}$, respectively) and the 10 per cent relative uncertainty due to spaxel variations. ${ }^{3}$

[C II] emission is clearly detected from the centre of NGC 185 (see Fig. 2, bottom left), while a more diffuse component appears to extend towards the east of the galaxy following the distribution of diffuse $\mathrm{HI}_{\mathrm{I}}$ and dust clouds (see Fig. 2, top panels). The peak of [C $\mathrm{II}]$ is located adjacent to the most massive dust and molecular gas clouds and coincides with the position of young stars emerging

\footnotetext{
${ }^{3}$ http://herschel.esac.esa.int/twiki/pub/Public/PacsCalibrationWeb/
} PacsSpectroscopyPerformanceAndCalibration_v2_4.pdf from the star-forming regions (see Fig. 2, bottom left). This suggests that the majority of $\left[\mathrm{C}_{\mathrm{II}}\right]$ emission arises from photodissociation regions (PDRs) positioned in between the star-forming regions and molecular gas reservoirs. The [O I] line is only detected in the very central region of NGC 185 (see Fig. 2, bottom right), coinciding with the peak in $[\mathrm{C} \mathrm{II}]$ emission and near the location of several young stars. Fig. 3 shows the [C II] $158 \mu \mathrm{m}$ (top) and [O I] $63 \mu \mathrm{m}$ (bottom) line profiles detected in the central spaxels of the PACS spectrometer.

\subsection{NRO 45 m CO(1-0) observations of NGC 205}

We observed the CO(1-0) line transition with the NRO $45 \mathrm{~m}$ telescope mapping the central and southern regions of NGC 205 (see 

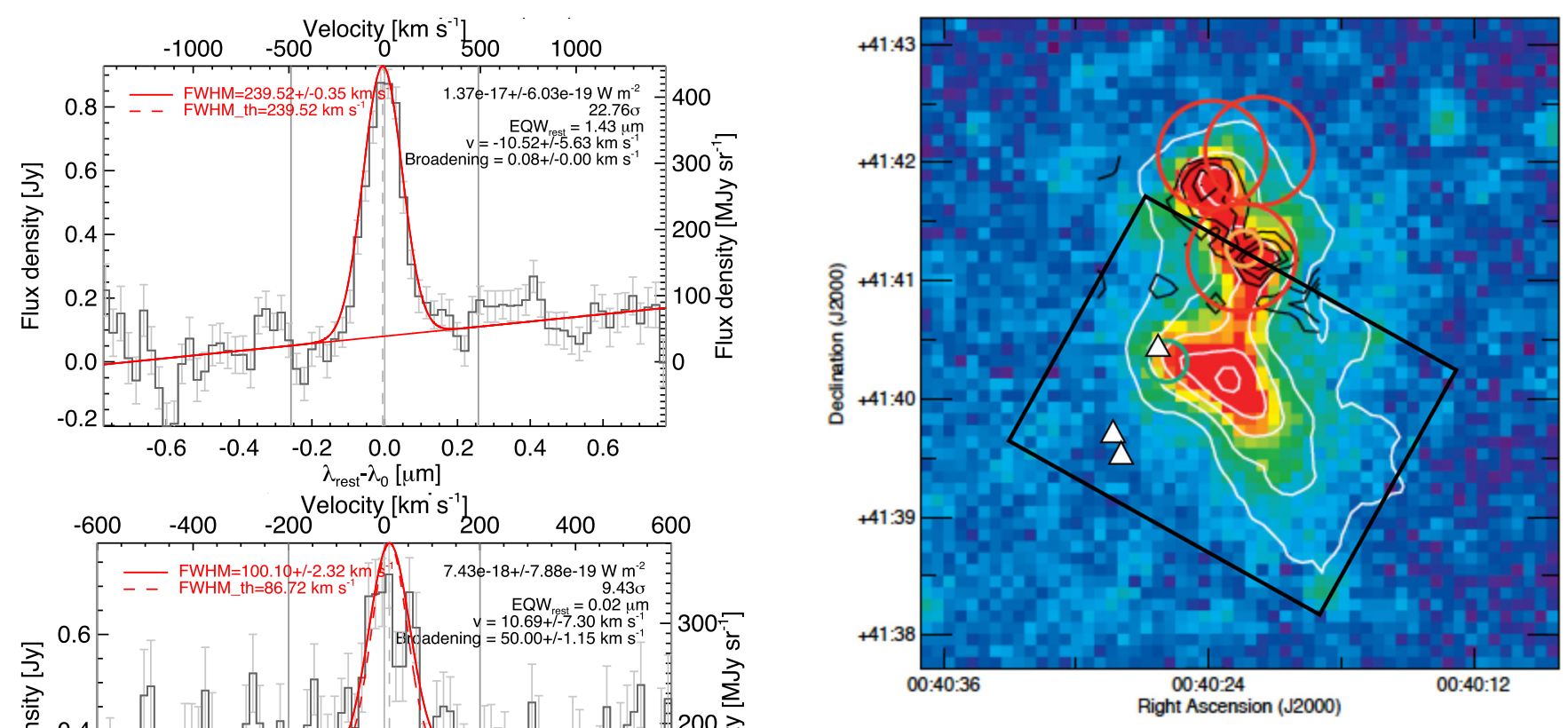

Figure 4. SPIRE $250 \mu \mathrm{m}$ image of NGC 205 overlaid with $\mathrm{H}_{\mathrm{I}}$ column density contours (Young \& Lo 1997, white solid lines) ranging from $2.6 \times$ $10^{20}$ to $1.9 \times 10^{21} \mathrm{~cm}^{-2}, \mathrm{H}_{2}$ column density contours (De Looze et al. 2012, black solid lines) ranging from $2.6 \times 10^{20}$ to $1.9 \times 10^{21} \mathrm{~cm}^{-2}$ in steps of 3.3 $\times 10^{20} \mathrm{~cm}^{-2}$. The red and green circles indicate the $\mathrm{CO}(1-0)$ pointings from Welch et al. (1998) and Young \& Lo (1996), respectively. The orange circle corresponds to the FWHM (18 arcsec) of the observed position with the JCMT at $1.1 \mathrm{~mm}$ (Fich \& Hodge 1991). The black box indicates the region covered by our NRO $45 \mathrm{~m}$ observations. The white triangles indicate Position 1 (top) and Positions 2A and 2B (bottom) where $\mathrm{CO}(1-0)$ line emission is detected from our new NRO $45 \mathrm{~m}$ data. This figure was taken from De Looze et al. (2012) and updated to include our recent CO observations.

(bottom) lines detected in NGC 185 with the PACS spectrometer in the central spaxel. The thin red lines indicate the first-order polynomial that was used to fit the continuum. The thick red lines represent the best-fitting Gaussian profile that was fit to the line emission. The dashed red lines indicate the best fit to the data assuming a linewidth similar to the spectral resolution of the instrument (i.e. $240 \mathrm{~km} \mathrm{~s}^{-1}$ for $\left[\mathrm{C}_{\text {II] }}\right.$ and $90 \mathrm{~km} \mathrm{~s}^{-1}$ for [O I $]$ ).

Fig. 4). We mapped a $3.2 \operatorname{arcmin} \times 2.7$ arcmin region with the On-The-Fly (OTF) mapping mode (Sawada et al. 2008) with a separation between scans of 5 arcsec. The observations were conducted during two separate runs in 2012, extending from January 22 until January 28 and April 16 until April 25. During both observing runs, the source IRC +30021 was used for pointing. The average wind speed during both observing runs was less than $5 \mathrm{~m} \mathrm{~s}^{-1}$ on average. The pointing was checked every hour, on average, and found to be accurate within 5 arcsec. The FWHM of the NRO $45 \mathrm{~m}$ beam at the $\mathrm{CO}(1-0)$ rest frequency of $115 \mathrm{GHz}$ is $16 \mathrm{arcsec}$ (which corresponds to about $64 \mathrm{pc}$ at the distance of NGC 205).

We observed the $\mathrm{CO}(1-0)$ line with the two sideband-separating (2SB) receivers $(\mathrm{T} 100 \mathrm{H}$ and $\mathrm{T} 100 \mathrm{~V})$ as front-end receivers (Nakajima et al. 2008). The analogue signal from T100 was converted to $4-8 \mathrm{GHz}$ before being transferred to the digital FX-type spectrometer SAM45 (Spectral Analysis Machine for the $45 \mathrm{~m}$ telescope). The back-end SAM45 was used with a frequency resolution of $488.24 \mathrm{kHz}$ that was rebinned to a spectral resolution of $1 \mathrm{MHz}$ or $2.6 \mathrm{~km} \mathrm{~s}^{-1}$. The typical system noise temperature during the observations ranged between $T_{\text {sys }} \sim 200$ and $300 \mathrm{~K}$, depending on the weather conditions. The total observing time during the different observing runs was $39 \mathrm{~h}$, with a total on-source time of $20 \mathrm{~h}$.

Data reduction was done with NOSTAR, which is a reduction tool for OTF observations developed by NRO. First of all, the data

with pointing errors greater than 5 arcsec were flagged and not used for the construction of the final map. Secondly, the image rejection ratio and the main beam efficiency were used to determine the absolute flux calibration following the method by Kerr et al. (2001). The uncertainty on the flux calibration is less than 15 per cent, which is based on the combined uncertainty from the measurement of the main beam efficiency and the daily variation of the image rejection ratio ( $\sim 5$ per cent during both observing runs). The antenna temperature $\left(T_{\mathrm{A}}^{*}\right)$ was converted to a main beam temperature $\left(T_{\mathrm{mb}}\right)$ using a main beam efficiency of $\eta_{\mathrm{mb}}=0.30-0.33^{4}$ and $T_{\mathrm{mb}}=T_{\mathrm{A}}^{*} / \eta_{\mathrm{mb}}$. The final data cube with a grid spacing of 7.5 arcsec was created by convolving with a Gaussian-tapered Bessel function:

$$
\frac{J_{1}(r / a)}{r / a} \times \exp \left[-\left(\frac{r}{b}\right)^{2}\right] \text {, with } a=1.55 / \pi \text { and } b=2.52 \text {, }
$$

with $J_{1}$ the first-order Bessel function and $r$ the distance between the data and grid point in a pixel. After convolution, the maps have an effective angular resolution of $19.3 \operatorname{arcsec}$ (or $77 \mathrm{pc}$ at the distance of NGC 205). Across our map, the average rms sensitivity ranged between $T_{\mathrm{rms}} \sim 15$ and $20 \mathrm{mK}$ at a velocity resolution of $2.6 \mathrm{~km} \mathrm{~s}^{-1}$.

We detect $\mathrm{CO}(1-0)$ line emission in three different positions. The line detections in Positions 1 and $2 \mathrm{~A}$ and $2 \mathrm{~B}$ are shown in Fig. 5. The line emission detected in Positions 2A and 2B is separated by only

\footnotetext{
${ }^{4}$ At the time of the observations, the main beam efficiency was $0.33 \pm 0.03$ and $0.30 \pm 0.02$ for the $\mathrm{T} 100 \mathrm{H}$ and $\mathrm{T} 100 \mathrm{~V}$ receivers, respectively.
} 

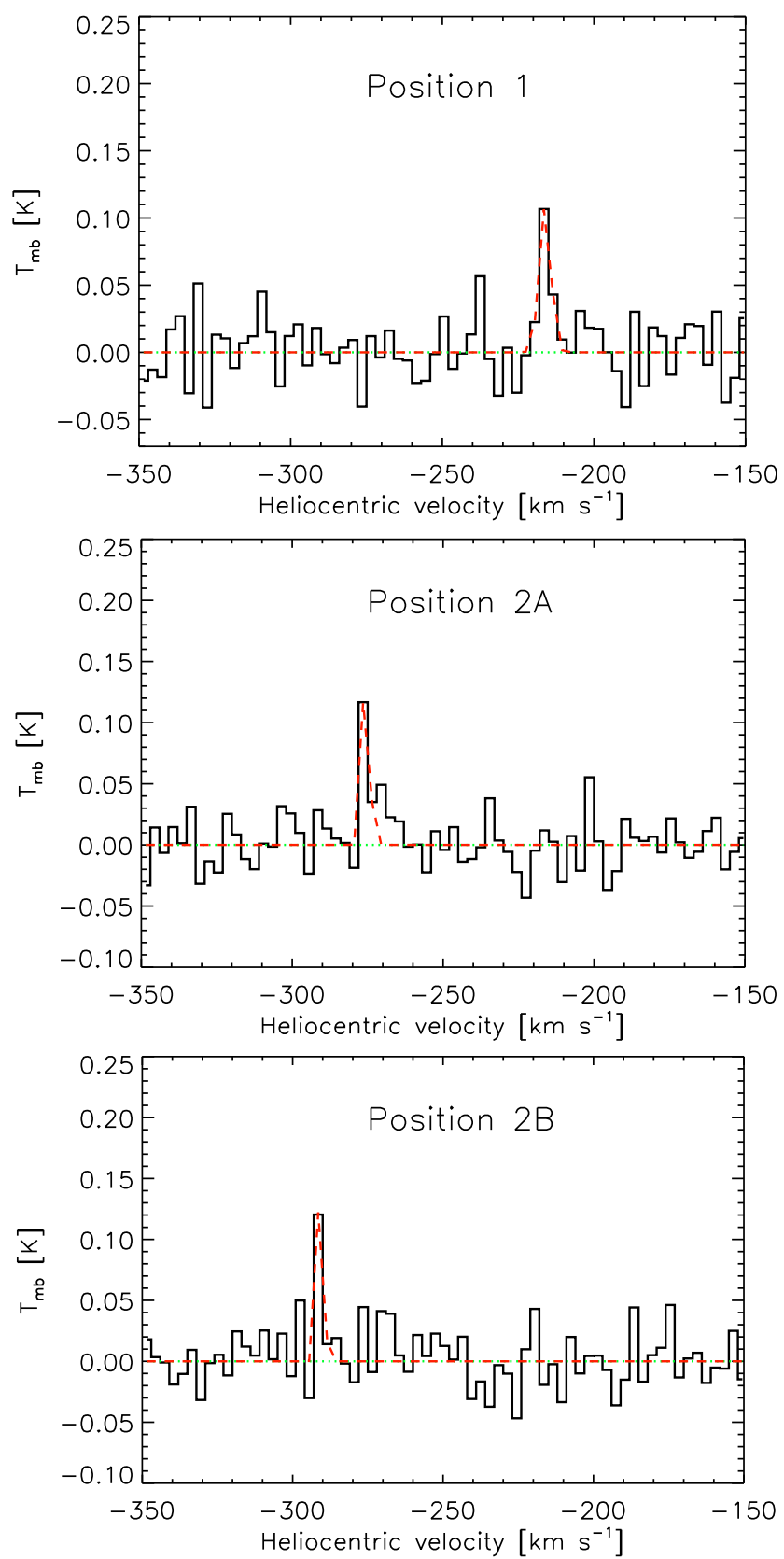

Figure 5. The $\mathrm{CO}(1-0)$ line profiles at the three positions (showing the average emission in a single pixel) where $\mathrm{CO}(1-0)$ line emission was detected in our NRO $45 \mathrm{~m}$ map covering the southern part of the galaxy NGC 205.

$\sim 15$ arcsec (similar to the size of the NRO $45 \mathrm{~m}$ beam at $115 \mathrm{GHz}$ ) and with peak velocities that are only $15 \mathrm{~km} \mathrm{~s}^{-1}$ apart, we cannot rule out that the two detections originate from the same cloud complex. We average the spectra of adjacent pixels with $\mathrm{CO}(1-0)$ detections to derive an average main beam temperature. We fit the baseline of the averaged spectra with a first-order polynomial, while the line emission is fit with a Gaussian profile. Table 5 gives an overview of the equatorial coordinates, central velocity $V$, linewidth FWHM, average main beam temperature $T_{\mathrm{mb}}$ and integrated line intensity $I_{\mathrm{CO}}$ of the three positions.

The detected $\mathrm{CO}(1-0)$ emission at Position 1 is located near the IRAM $\mathrm{CO}(1-0)$ detection (with a $\sim 3 \sigma$ significance) reported by Young \& Lo (1996) at a position (RA, Dec.) $=\left(0^{\mathrm{h}} 40^{\mathrm{m}} 25^{\mathrm{s}} .9\right.$, $\left.+41^{\circ} 40^{\prime} 19^{\prime \prime}\right)$. While the central velocity $\left(-218.8 \pm 1.8 \mathrm{~km} \mathrm{~s}^{-1}\right)$ reported by Young \& Lo (1996) is in fair agreement with our NRO $45 \mathrm{~m} \mathrm{CO}(1-0)$ observations $(-216.1 \pm 0.6)$, we find a smaller linewidth $\left(4.5 \pm 1.1 \mathrm{~km} \mathrm{~s}^{-1}\right)$ compared to the values reported by Young \& Lo (1996) $\left(\mathrm{FWHM}=19.8 \pm 3.7 \mathrm{~km} \mathrm{~s}^{-1}\right)$. This difference between both $\mathrm{CO}(1-0)$ observations can likely be attributed to the IRAM $30 \mathrm{~m}$ beam (FWHM $\sim 21 \mathrm{arcsec}$ ) being offset by about 7 arcsec from the peak $\mathrm{CO}(1-0)$ line emission, which might not have picked up the peak of line emission. The central velocity $\left(-215 \mathrm{~km} \mathrm{~s}^{-1}\right)$ and linewidth $\left(\sim 10 \mathrm{~km} \mathrm{~s}^{-1}\right)$ observed for H I clouds at the same position are in good agreement with the NRO $45 \mathrm{~m}$ $\mathrm{CO}(1-0)$ observations. Also, the linewidth $\left(6.6 \pm 0.7 \mathrm{~km} \mathrm{~s}^{-1}\right)$ of the CO emission detected with IRAM in the north of NGC 205 is more consistent with the line-width measurement from our NRO 45 m observations.

The $\mathrm{CO}(1-0)$ emission detected at Position 2A has an average line intensity similar to the line emission detected at Position 1 and is centred around a heliocentric velocity of $-275.5 \mathrm{~km} \mathrm{~s}^{-1}$, with a linewidth $\left(2.5 \pm 0.6 \mathrm{~km} \mathrm{~s}^{-1}\right)$ that is similar to our spectral resolution. The adjacent $\mathrm{CO}(1-0)$ detection at Position $2 \mathrm{~B}$ is centred around a heliocentric velocity of $-290.8 \mathrm{~km} \mathrm{~s}^{-1}$ and has a similar narrow linewidth of $2.4 \pm 0.9 \mathrm{~km} \mathrm{~s}^{-1}$. The central velocities of these $\mathrm{CO}$ clouds are at the limit of the stellar velocities (ranging between -280 and $-140 \mathrm{~km} \mathrm{~s}^{-1}$; Geha et al. 2010) and outside of the Hi velocity range ( -260 to $-140 \mathrm{~km} \mathrm{~s}^{-1}$ ) for NGC 205 (Young \& Lo 1997). We note that the central velocity of the cloud is far from the range of $\mathrm{H}_{\mathrm{I}}$ velocities detected in our Galaxy $(-130$ to $45 \mathrm{~km} \mathrm{~s}^{-1}$; Braun et al. 2009) and this emission does not belong to our Galaxy. The disturbed nature of the distribution of atomic and molecular clouds in NGC 205 (Young \& Lo 1997) and the offset from the main stellar body in NGC 205 suggests that the gas clouds have not yet settled into a stable configuration. The irregular disposition of this molecular gas cloud might be the result of a recent tidal interaction that has disturbed the gas distribution in NGC 205. Alternatively, the $\mathrm{CO}(1-0)$ line emission at Position $2 \mathrm{~B}$ might be a false detection and rather correspond to a noise peak, given the small linewidth that resembles the spectral resolution of the observations. The line emission detected in Position 2A is unlikely to correspond to instrumental noise, given its detection in several adjacent pixels (corresponding to $1.5 \times$ FWHM), although we cannot entirely rule out that it corresponds to a local noise peak.

The line emission in our NRO 45 m map of NGC 205 is detected in only 2-7 adjacent 7.5 arcsec $\times 7.5$ arcsec sized pixels, indicating that the size of molecular clouds in NGC 205 is extremely small with typical values of $\lesssim 20-25$ arcsec (or 80-100 pc). Interferometric $\mathrm{CO}(1-0)$ observations with the Berkeley-Illinois-Maryland Association (BIMA) array (with a $40 \mathrm{pc} \times 20 \mathrm{pc}$ beam) can barely resolve molecular clouds and measure a cloud size around 40-60 pc for a giant molecular cloud in the north of NGC 205 (Young \& Lo 1996). Based on a cloud size of 40-60 pc, the first Larson scaling relation between a cloud's size and velocity dispersion (Larson 1981) predicts a linewidth of $4.5-5.2 \mathrm{~km} \mathrm{~s}^{-1}$. The similarity with the observed linewidths $\left(2.5-4.4 \mathrm{~km} \mathrm{~s}^{-1}\right)$ suggests that the clouds are virialized and experience very little internal gas turbulence.

We derive molecular gas masses $M_{\mathrm{H}_{2}}=A N_{\mathrm{H}_{2}} m_{\mathrm{H}_{2}}$, where $A$ is the surface of the CO-emitting region (cf. the number of detected pixels in every position indicated in Table 2), $m_{\mathrm{H}_{2}}$ is the molecular hydrogen mass and the column density of $\mathrm{H}_{2}$ is calculated as

$N_{\mathrm{H}_{2}}=X_{\mathrm{CO}} I_{\mathrm{CO}(1-0)}$ 
Table 2. Overview of the CO line fitting parameters with the position coordinates (of the brightest pixel), average main beam temperature, velocity, linewidth and integrated CO line intensity for every position. The last two columns present the molecular gas masses derived from the average integrated $\mathrm{CO}$ line intensity summed over all detected pixels based on Galactic and metallicity-dependent $X_{\mathrm{CO}}$ factors.

\begin{tabular}{llccccccc}
\hline Position & $\begin{array}{l}\text { RA (J2000) } \\
(\mathrm{h} \mathrm{m} \mathrm{s})\end{array}$ & $\begin{array}{c}\text { Dec. (J2000) } \\
\left({ }^{\prime \prime \prime \prime}\right)\end{array}$ & $\begin{array}{c}T_{\mathrm{mb}} \\
(\mathrm{K})\end{array}$ & $\begin{array}{c}v_{\mathrm{cen}} \\
\left(\mathrm{km} \mathrm{s}^{-1}\right)\end{array}$ & $\begin{array}{c}\mathrm{FWHM} \\
\left(\mathrm{km} \mathrm{s}^{-1}\right)\end{array}$ & $\begin{array}{c}I_{\mathrm{CO}} \\
\left(\mathrm{K} \mathrm{km} \mathrm{s}^{-1}\right)\end{array}$ & $\begin{array}{c}N_{\mathrm{pix}} \\
M_{\mathrm{H}_{2}}\left(X_{\mathrm{CO}, \mathrm{Gal}}\right) \\
\left(\mathrm{M}_{\odot}\right)\end{array}$ & $\begin{array}{c}M_{\mathrm{H}_{2}}\left(X_{\mathrm{CO}, \mathrm{Met}}\right) \\
\left(\mathrm{M}_{\odot}\right)\end{array}$ \\
\hline Position 1 & $00: 40: 26.0$ & $+41: 40: 36.4$ & $0.11 \pm 0.02$ & $-216.1 \pm 0.6$ & $4.5 \pm 1.1$ & 0.49 & 7 & $1.0 \times 10^{4}$ \\
Position 2A & $00: 40: 27.4$ & $+41: 39: 28.9$ & $0.18 \pm 0.07$ & $-275.5 \pm 0.3$ & $2.5 \pm 0.6$ & 0.46 & 6 & $0.8 \times 10^{4}$ \\
Position 2B & $00: 40: 28.0$ & $+41: 39: 43.9$ & $0.16 \pm 0.07$ & $-290.8 \pm 0.5$ & $2.4 \pm 0.9$ & 0.37 & 2 & $0.2 \times 10^{4}$ \\
\hline
\end{tabular}

Table 3. Overview of masses for the different ISM components derived from various observational constraints. The different gas mass measurements are discussed in Section 4. The dust masses are taken from De Looze et al. (2016) for NGC 147 and NGC 185 and from De Looze et al. (2012) for NGC 205. The total gas mass is obtained by summing the gas masses from different gas phases, scaled by a factor of 1.36 to include helium, using a Galactic and $Z$-dependent $X_{\mathrm{CO}}$ conversion factor. The gas-to-dust mass ratios are derived from the global gas and dust mass measurements. The values between parentheses are derived based on $H$-band luminosity-dependent $X_{\mathrm{CO}}$ conversion factors.

\begin{tabular}{lccc}
\hline Galaxy & NGC 147 & NGC 185 & NGC 205 \\
\hline$M_{\mathrm{H}_{\mathrm{I}}}\left(10^{4} \mathrm{M}_{\odot}\right)$ & $\leq 0.4$ & 11 & 40 \\
$M_{\mathrm{H}_{2}, \mathrm{Gal}}\left(10^{4} \mathrm{M}_{\odot}\right)$ & $\leq 1.3$ & 2.8 & 23 \\
$M_{\mathrm{H}_{2}, Z}\left(10^{4} \mathrm{M}_{\odot}\right)$ & $\leq 15.8$ & 29.1 & 144 \\
$M_{\text {ion }}\left(\mathrm{M}_{\odot}\right)$ & - & 1 & $\leq 1$ \\
Warm $M_{\mathrm{H}_{2}}\left(10^{2} \mathrm{M}_{\odot}\right)$ & - & 2.4 & - \\
Hot X-ray gas $\left(10^{4} \mathrm{M}_{\odot}\right)$ & $\leq 0.2$ & $\leq 0.2$ & $\leq 3.8$ \\
Total $M_{\text {gas, Gal }}\left(10^{4} \mathrm{M}_{\odot}\right)$ & $\leq 2.7$ & 18.8 & 85.7 \\
Total $M_{\text {gas, } Z}\left(10^{4} \mathrm{M}_{\odot}\right)$ & $\leq 22.4$ & 54.5 & 250.2 \\
$M_{\text {dust }}\left(10^{3} \mathrm{M}_{\odot}\right)$ & $\leq 0.128$ & 5.1 & $11-18$ \\
GDR $\left(\mathrm{Gal} . \mathrm{X}_{\mathrm{CO}}\right)$ & - & 37 & 48 \\
GDR $\left(Z\right.$-dep. $\left.\mathrm{X}_{\mathrm{CO}}\right)$ & - & 107 & 139 \\
\hline
\end{tabular}

with $I_{\mathrm{CO}(1-0)}$ the integrated main beam line intensity in units of $\mathrm{K}$ $\mathrm{km} \mathrm{s}^{-1}$ and $X_{\mathrm{CO}}$ the conversion factor. Since the $X_{\mathrm{CO}}$ scaling factor might depend on metallicity, we apply the usual Galactic scaling factor $\left(X_{\mathrm{CO}}=2.0 \times 10^{20} \mathrm{~cm}^{-2}\left[\mathrm{~K} \mathrm{~km} \mathrm{~s}^{-1}\right]^{-1}\right.$; Strong \& Mattox 1996; Dame, Hartmann \& Thaddeus 2001; Ackermann et al. 2011) as well as a $H$-band luminosity-dependent conversion factor $\left(X_{\mathrm{CO}}\right.$ $=12.5 \times 10^{20} \mathrm{~cm}^{-2}\left[\mathrm{~K} \mathrm{~km} \mathrm{~s}^{-1}\right]^{-1}$ ) following the prescriptions from Boselli et al. (2002). The $H$-band luminosity of a galaxy is shown to scale with the abundance of metals and can be considered as a metallicity-dependent $X_{\mathrm{CO}}$ factor. We derive molecular gas masses of $M_{\mathrm{H}_{2}}=1.0-6.2 \times 10^{4}, 0.8-5.0 \times 10^{4}$ and $0.2-1.3 \times$ $10^{4} \mathrm{M}_{\odot}$ for Positions $1,2 \mathrm{~A}$ and $2 \mathrm{~B}$, respectively, within the limits of the two different conversion factors. A comparison with previous $\mathrm{CO}(1-0)$ observations of the north and central regions of NGC 205 (see Section 4.1) show that the molecular gas clouds in the south account for only 1/10th of the total molecular gas reservoir in NGC 205.

Total molecular gas masses (see Table 3) using a metallicitydependent $X_{\mathrm{CO}}$ factor are more than three times higher compared to the atomic gas mass in NGC $205\left(M_{\mathrm{H}_{\mathrm{I}}}=4 \times 10^{5} \mathrm{M}_{\odot}\right)$, which seems unrealistic given the low SF activity in NGC 205. The choice of a Galactic $X_{\mathrm{CO}}$ factor is also consistent with the Galactic conversion factor derived by Bolatto et al. (2008) for a molecular cloud in the centre of NGC 205 based on the virial mass assumptions. Molecular gas mass depletion factors ( $\log \tau_{\mathrm{H}_{2}} \sim 0.6-0.8 \mathrm{Gyr}$ ) based on $H$-band luminosity-dependent $X_{\mathrm{CO}}$ factors would, however, better agree with the trend between molecular gas depletion time-scale and specific

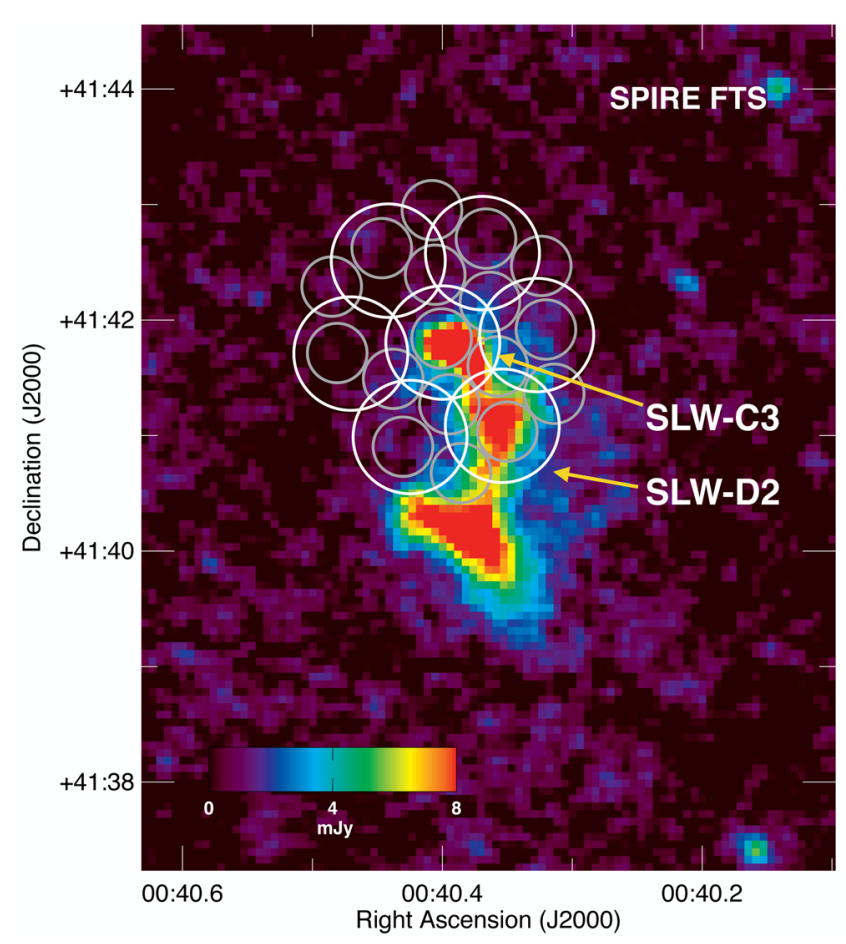

Figure 6. PACS $160 \mu \mathrm{m}$ image of NGC 205 overlaid with the SPIRE FTS pointing targeting the $\mathrm{CO}$ peak in the north with individual jiggle positions for the SSW and SLW detectors indicated as grey and white circles, respectively. The SPIRE FTS SLW-C3 and SLW-D2 bolometers are labelled in the figure.

star formation rate $\left(\mathrm{sSFR}=\mathrm{SFR} / \mathrm{M}_{\star}\right.$ ) observed for the COLD GASS sample (Saintonge et al. 2011). To account for uncertainties on the $X_{\mathrm{CO}}$ factor, we will mention molecular gas masses derived from both Galactic and $H$-band luminosity-dependent $X_{\mathrm{CO}}$ factors in the remainder of this work.

\subsection{SPIRE FTS spectroscopy data of NGC 205}

Herschel observations of NGC 205 were acquired as part of the Guaranteed Time program 'Very Nearby Galaxies Survey' (PI: C. Wilson). The SPIRE FTS spectra were obtained in sparse spatial sampling and high-spectral resolution mode, covering the 194$671 \mu \mathrm{m}$ wavelength range. One single pointing targeting the $\mathrm{CO}$ peak in the north of NGC 205 (see Fig. 6) was observed with 74 repetitions. The 35 detectors of the SSW (SPIRE Short Wavelength) array covered the 194-313 $\mu \mathrm{m}$ range, while the SLW (SPIRE Long Wavelength) array of 19 detectors covered the 303-671 $\mu \mathrm{m}$ wavelength range. The SSW and SLW arrays have an average FWHM of 19 and 34 arcsec, respectively (Makiwa et al. 2013).

The SPIRE FTS data were reduced in HIPE v14.0.0, with version SPIRE_CAL_14_2 of the calibration files. The standard pipeline in 
HIPE for single pointing SPIRE spectrometer observations was used for data reduction, assuming a point source calibration without apodization. The standard pipeline included a first- and secondorder deglitching procedure, non-linearity and phase corrections, baseline subtraction and corrections for the telescope and instrument emission. We fit spectral lines in the SPIRE FTS data using the SPIRE Spectrometer Line Fitting algorithm in HIPE. All emission lines of interest (i.e. $\mathrm{CO}$ line transitions from ${ }^{12} \mathrm{CO}(4-3)$ up to ${ }^{12} \mathrm{CO}(13-12)$ and the two [C $\left.\mathrm{I}\right]$ line and [N II] $205 \mu \mathrm{m}$ line transitions) were fit simultaneously using a third-order polynomial for the continuum, while the line profile is fit with a sinc function. We assume the line profiles have a width that corresponds to the spectral resolution of the instrument (ranging from 280 to $970 \mathrm{~km} \mathrm{~s}^{-1}$ towards longer wavelengths). Based on the typical linewidths ( $\lesssim 20 \mathrm{~km} \mathrm{~s}^{-1}$; Young \& Lo 1996, 1997) for the observed $\mathrm{HI}_{\mathrm{I}}$ and CO line transitions in NGC 205, we are confident that the line profiles will be set by the instrument's spectral imprint.

We do not detect $\mathrm{CO},\left[\mathrm{C}_{\mathrm{I}}\right]$ or $[\mathrm{N}$ II] emission in any of the SPIRE FTS bolometers. The $1 \sigma$ upper limits derived from the line fitting algorithm for the spectra of the SPIRE SLW-C3 bolometer constrains the $\left[\mathrm{C}_{\mathrm{I}}\right]$ integrated line fluxes in the CO-peak in the north $\left(I_{\left[\mathrm{C}_{1}\right] 1-0} \leq 1.06 \times 10^{-18} \mathrm{~W} \mathrm{~m}^{-2}\right.$ or $65 \mathrm{Jy} \mathrm{km} \mathrm{s}^{-1} ; I_{[\mathrm{CI}] 2-1} \leq 1.09 \times$ $10^{-18} \mathrm{~W} \mathrm{~m}^{-2}$ or $40 \mathrm{Jy} \mathrm{km} \mathrm{s}^{-1}$ ) and the spectra of the SPIRE SLWD2 bolometer similarly constrain $\left[\mathrm{C}_{\mathrm{I}}\right]$ integrated line fluxes in the centre of NGC $205\left(I_{[\mathrm{Ci}] 1-0} \leq 1.04 \times 10^{-18} \mathrm{~W} \mathrm{~m}^{-2}\right.$ or $63 \mathrm{Jy} \mathrm{km} \mathrm{s}^{-1}$; $I_{\left[\mathrm{C}_{1}\right] 2-1} \leq 1.07 \times 10^{-18} \mathrm{~W} \mathrm{~m}^{-2}$ or $\left.40 \mathrm{Jy} \mathrm{km} \mathrm{s}^{-1}\right)$. We furthermore use the $1 \sigma$ upper limits on the $[\mathrm{N} \mathrm{II}] 205 \mu \mathrm{m}$ flux $\left(I_{[\mathrm{NII}}\right]_{1-0} \leq 8.08 \times$ $10^{-19} \mathrm{~W} \mathrm{~m}^{-2}$ ) in the centre of NGC 205 to constrain the [C II] contribution from ionized gas in Section 3.1.

\subsection{Ancillary data}

For NGC147, we only have $\mathrm{H}$ I and CO observations to constrain the gas mass. We rely on the upper gas mass limits from $\mathrm{H}$ I and $\mathrm{H}_{2}$ observations reported by Young \& Lo (1997) and Sage et al. (1998), respectively, to constrain the gas content in NGC 147.

For NGC185, we have $\mathrm{H}$ I, CO and $\mathrm{H} \alpha$ observations and Spitzer Infrared Spectrograph (IRS) spectra with rotational $\mathrm{H}_{2}$ lines to constrain the atomic, cold molecular, ionized and warm molecular gas masses. We reduced HI data for NGC 185 observed with the Very Large Array in configurations $\mathrm{C}(8 \mathrm{~h})$ and $\mathrm{D}(4.5 \mathrm{~h})$, in a similar way as presented in Young \& Lo (1997). We derive a moment-0 map of the $\mathrm{H}_{\mathrm{I}}$ observations following the same strategy as Young \& Lo (1997). The H I map was derived at medium resolution of 28 arcsec $\times 26 \operatorname{arcsec}($ or $84 \mathrm{pc} \times 78 \mathrm{pc}$ ) with rms noise level of $0.54 \mathrm{mJy}$ beam $^{-1}$ (or $0.46 \mathrm{~K}$ ), which corresponds to a H i column density of $\sim 3 \times 10^{19} \mathrm{~cm}^{-2}$. At the medium resolution of $28 \operatorname{arcsec} \times 26 \operatorname{arcsec}$, we observe a peak in $\mathrm{H}_{\mathrm{I}}$ column density $N_{\mathrm{H}_{\mathrm{I}}} \sim 3.1 \times 10^{20} \mathrm{~cm}^{-2}$. Assuming optically thin $\mathrm{H}_{\mathrm{I}}$ emission, we derive a total $\mathrm{H}_{\mathrm{I}}$ mass of $M_{\mathrm{HI}}=1.1 \times 10^{5} \mathrm{M}_{\odot}$, scaled to our adopted distance $\mathrm{D}=$ $0.616 \mathrm{Mpc}$. We, furthermore, use the $\mathrm{CO}$ map obtained from interferometric observations with the BIMA array presented by Young (2001). For the analysis in this paper, we use the CO intensity map with $5.5 \operatorname{arcsec} \times 4.6 \operatorname{arcsec}(17 \mathrm{pc} \times 14 \mathrm{pc})$ resolution and an rms noise level of $0.070 \mathrm{Jy}^{\text {beam }}{ }^{-1}$ (or $0.25 \mathrm{~K}$ ).

For NGC 205, we have $\mathrm{H}$ I, $\mathrm{CO}$ and $\mathrm{H} \alpha$ observations to constrain the atomic, cold molecular and ionized gas masses. The CO observations in the north and centre of NGC 205 (Young \& Lo 1996; Welch et al. 1998) are complemented with our new NRO $45 \mathrm{~m}$ observations covering the southern regions of NGC 205 (see Section 2.2). We furthermore have Herschel SPIRE FTS spectra with [C $\mathrm{I}]$ line transitions that allow us to constrain the CO-dark molecular gas content in NGC 205. The ancillary H I and JCMT CO(3-2) data sets used for NGC 205 were described in De Looze et al. (2012).

\section{PHYSICAL GAS CHARACTERISTICS}

\subsection{Origin of the $[\mathrm{C} \mathrm{II}]$ emission}

With an ionization potential of $11.3 \mathrm{eV}$, the [C $\mathrm{II}]$ line emission in NGC 185 can originate from PDRs, the cold neutral medium (CNM) and ionized gas phases. The $[\mathrm{C}$ II] contribution of the ionized gas phase is considered to be negligible in NGC 185 , given the weak emission of ionized gas tracers (e.g. $\mathrm{H} \alpha$ ) and the absence of a strong radiation field (De Looze et al. 2016). The latter argument is also supported by the negligible [ $\left.\mathrm{C}_{\mathrm{II}}\right]$ contribution from ionized gas in NGC 205 assuming that its ISM conditions are similar to NGC 185. The $\left[\mathrm{C}_{\mathrm{II}}\right]$ contribution from ionized gas is estimated in NGC 205 based on the $1 \sigma$ upper limit on its [ $\mathrm{N}_{\text {II }} 205 \mu \mathrm{m}$ emission as observed with the SPIRE FTS instrument onboard Herschel (see Section 2.3). Theoretical models predict the line ratios of $\left[\mathrm{C}_{\mathrm{II}}\right] /[\mathrm{N} \mathrm{II}]_{205} \sim 3-4$ for a range of different electron densities (Oberst et al. 2006). Based on the lower limit on the observed $[\mathrm{C} \mathrm{II}] /[\mathrm{N} \mathrm{II}]_{205}$ line ratio ([C $\mathrm{II}] /[\mathrm{N} \text { II }]_{205} \geq 123$ ) in NGC 205 , we estimate a maximum [C $\left.\mathrm{CI}\right]$ contribution of the ionized gas phase of $\leq 4$ percent. To predict the $\left[\mathrm{C}_{\mathrm{II}}\right]$ emission produced in the CNM excited by collisions with hydrogen atoms or molecules, we apply equation 1 from Madden et al. (1997):

$I_{[\mathrm{C} \text { II }}=2.35 \times 10^{-21} N_{\mathrm{C}+}\left(\frac{2 \times \exp (-91.3 / T)}{1+2 \times \exp (-91.3 / T)+\left(n_{\text {crit }} / n_{\mathrm{H}}\right)}\right)$,

with $I_{\left[\mathrm{C}_{\text {II] }}\right.}$ the $\left[\mathrm{C}_{\mathrm{II}}\right]$ intensity in units of $\mathrm{erg} \mathrm{s}^{-1} \mathrm{~cm}^{-2} \mathrm{sr}^{-2}$ and $N_{\mathrm{C}+}$ the $\mathrm{C}^{+}$column density calculated as $N_{\mathrm{C}+}=X_{\mathrm{C}+} N_{\mathrm{H}_{\mathrm{I}}}$ with $N_{\mathrm{H}_{\mathrm{I}}}$ the H I column density. We assume a gas temperature $T=50 \mathrm{~K}$ and gas density $n_{\mathrm{H}}=100 \mathrm{~cm}^{-3}$ typical for the CNM (Madden et al. 1997). The $\mathrm{C}^{+} / \mathrm{H}$ abundance $\left(X_{\mathrm{C}^{+}} \sim 5.0 \times 10^{-5}\right)$ is scaled relative to the solar carbon abundance $\left(X_{\mathrm{C}} \sim 1.4 \times 10^{-4}\right.$; Sofia et al. 1997) based on the metallicity of NGC $185\left(0.36 \mathrm{Z}_{\odot}\right.$, see Section 1.3$)$ under the assumption $^{5}$ that all of the carbon is in the form of $\mathrm{C}^{+}$. We assume a critical density $n_{\text {crit }}(\mathrm{H}) \sim 1.6 \times 10^{3} \mathrm{~cm}^{-3}$ (Goldsmith et al. 2012) for collisions with $\mathrm{H}$ atoms.

We convolve the observed $[\mathrm{C} \mathrm{II}]$ map to the resolution of the $\mathrm{HI}$ observations $(\sim 28 \operatorname{arcsec})$ to compare the observed $\left[\mathrm{C}_{\mathrm{II}}\right]$ intensity with the predicted contribution from the CNM. We find [C $\mathrm{CI}]$ contributions ranging from 12 per cent to 25 per cent, implying that only up to a quarter of the $[\mathrm{C} \mathrm{II}]$ emission originates from the CNM. The highest contributions from $\mathrm{H}_{\mathrm{I}}$ clouds to the $[\mathrm{C}$ II] emission occur in the more diffuse emission regions, while the $[\mathrm{C} I \mathrm{II}]$ contribution from the CNM reaches a minimum in the dust mass peak south-west of the centre. The small contribution from H i clouds suggests that the majority of $[\mathrm{C} \mathrm{II}]$ emission in NGC 185 originates from PDRs.

Based on the total integrated CO flux density reported by Young (2001) $\left(S_{\mathrm{CO}} \sim 8.8 \mathrm{Jy} \mathrm{km} \mathrm{s}^{-1}\right)$ and the sum of the $\geq 3 \sigma$ regions in the $\left[\mathrm{C}_{\mathrm{II}}\right] \operatorname{map}\left(F_{[\mathrm{C} \text { II] }}\right.$ (total $\left.)=1.33 \times 10^{-16} \mathrm{~W} \mathrm{~m}^{-2}\right)$, we derive a $\left[\mathrm{C}\right.$ II]/CO ratio of $\sim 3.9 \times 10^{3}$. The line ratio in NGC 185 is higher compared to the $\left[\mathrm{C}_{\mathrm{II}}\right] / \mathrm{CO}$ ratio observed in NGC $205\left(\sim 1.9 \times 10^{3}\right.$;

\footnotetext{
${ }^{5}$ This assumption might not be appropriate given the soft radiation field and low gas temperature $T \sim 40-70 \mathrm{~K}$ in NGC 185, which might prevent the ionization of carbon and/or excitation of $\mathrm{C}^{+}$. For lower $\mathrm{C}^{+}$abundances, the $\mathrm{CNM}$ contribution to the $[\mathrm{C} \mathrm{II}]$ emission will be smaller than the values quoted here.
} 
De Looze et al. 2012), similar to the range of values observed in starburst galaxies (Stacey et al. 1991; Negishi et al. 2001), but at the low end of the line ratios observed in low-metallicity star-forming dwarf galaxies, ranging from 4000 to 80000 (Madden 2000; Cormier et al. 2010; Madden, Cormier \& Remy-Ruyer 2016). In these low-metallicity star-forming dwarf galaxies, CO molecules are more easily photodissociated due to the hard radiation and a porous ISM structure, leaving behind a layer of self-shielding $\mathrm{H}_{2}$ that is not traced by the $\mathrm{CO}$ observations. Based on the low [C II]/CO line ratios in dSphs, this CO-dark gas component (Wolfire, Hollenbach \& McKee 2010) is expected to be significantly less important in NGC 185 and NGC 205 compared to the CO-dark gas reservoir in metal-poor star-forming dwarfs. The UV radiation fields in these dSphs is several times weaker compared to star-forming dwarf galaxies, enabling the $\mathrm{CO}$ molecules to survive and trace the bulk of $\mathrm{H}_{2}$ gas mass.

\subsection{Photoelectric efficiency}

A map of the total-infrared (TIR) emission in NGC 185 is calculated based on the MIPS $24 \mu \mathrm{m}$, PACS $100 \mu \mathrm{m}$ and PACS $160 \mu \mathrm{m}$ maps and the prescriptions of Galametz et al. (2013). For the computation of the TIR emission, all maps have been convolved to the resolution of the PACS $160 \mu \mathrm{m}$ waveband with the appropriate kernels from Aniano et al. (2011). To measure line intensities, we similarly convolve all line maps to the resolution of the PACS $160 \mu \mathrm{m}$ waveband. Since the $\left[\mathrm{O}_{\mathrm{I}}\right]$ line is only detected in the centre of NGC 185, we measure fluxes $\left(F_{[\mathrm{C} \text { II] }}=3.5 \pm 0.3 \times 10^{-17} \mathrm{~W} \mathrm{~m}^{-2}, F_{\left[\mathrm{O}_{\mathrm{I}}\right]}=8.6 \pm\right.$ $\left.5.0 \times 10^{-18} \mathrm{~W} \mathrm{~m}^{-2}, F_{\mathrm{TIR}}=2.4 \pm 0.1 \times 10^{-15} \mathrm{~W} \mathrm{~m}^{-2}\right)^{6}$ within a circular aperture of radius $R=12.1$ arcsec (or similar to the FWHM of the PACS $160 \mu \mathrm{m}$ beam) towards the centre of NGC 185. Since [O I ] emission is only detected in the central spaxel, we can also measure the total $\left[\mathrm{O}_{\mathrm{I}}\right]$ flux $\left(F_{\left[\mathrm{O}_{\mathrm{I}}\right]}=10.6 \pm 0.8 \times 10^{-18} \mathrm{~W} \mathrm{~m}^{-2}\right)$ by applying a point-source correction to the flux detected in the central spaxel. Given that the uncertainty on the corrected flux measurement from the central spaxel is lower, we will use the latter [O I] flux measurement in the remainder of this work.

Based on these line measurements for the central region, we derive $\left[\mathrm{C}_{\mathrm{II}}\right] / \mathrm{TIR}=0.015 \pm 0.004$ and $\left[\mathrm{C}_{\mathrm{II}}\right]+\left[\mathrm{O}_{\mathrm{I}}\right] / \mathrm{TIR}=0.021$ \pm 0.004 line ratios. The line ratios are indicative of the efficiency of the photoelectric effect, in case the [C $\mathrm{II}]$ and [O I] line emission is a good proxy for the gas cooling (and thus gas heating) and TIR is representative of the energy of stars that goes into heating the dust. With ratios higher than 1 per cent, the photoelectric heating of neutral gas in the central regions of NGC 185 is considered more efficient compared to the average $\left[\mathrm{C}_{\mathrm{II}}\right] / \mathrm{TIR}$ ratios $(0.1-1$ per cent) in normal star-forming galaxies (e.g. Malhotra et al. 2001; Brauher, Dale \& Helou 2008; Smith et al. 2016). Given the soft radiation field in NGC 185 and the bright features of polycyclic aromatic hydrocarbons (PAHs) detected in the Spitzer IRS spectra (Marleau et al. 2010), the high photoelectric efficiency might be attributed to a high PAH abundance and/or a low fraction of grain charging. Similarly high photoelectric efficiencies were observed in a sample of low-surface brightness dwarf galaxies (Cigan et al. 2016).

\footnotetext{
${ }^{6}$ The uncertainty on the TIR emission accounts for the observational uncertainties on the MIPS $24 \mu \mathrm{m}$ and PACS measurements and the scatter in the calibrations of Galametz et al. (2013).
}

\subsection{PDR diagnostics}

To examine the state of the gas in NGC 185, the observed [C $\mathrm{II}$ ] and $\left[\mathrm{O}_{\mathrm{I}}\right.$ ] line and TIR emission are compared to PDR models using the PDR toolbox (Pound \& Wolfire 2008). For a comparison of the observed $\left[\mathrm{O}_{\mathrm{I}}\right] /\left[\mathrm{C}_{\mathrm{II}}\right]$ and $\left(\left[\mathrm{C}_{\mathrm{II}}\right]+\left[\mathrm{O}_{\mathrm{I}}\right] / \mathrm{TIR}\right)$ line ratios to PDR models, we assume that all of the $\left[\mathrm{C}_{\mathrm{II}}\right]$ and $\left[\mathrm{O}_{\mathrm{I}}\right]$ emission originates from PDRs. The line ratios in the PDR toolbox are calculated for a plane-parallel geometry with elemental abundances and grain properties fixed for a metallicity $1 \mathrm{Z}_{\odot} \cdot{ }^{7}$ Due to the assumption in the PDR models of a slab geometry that is illuminated and emitting on one side, we need to make certain corrections to the observed line emission before comparing it to PDR models. We apply a similar strategy to correct our observations as followed by Parkin et al. (2013, 2014) and Hughes et al. (2015). Since we observe the front and back side emission of clouds in NGC 185 (under the assumption of optically thin IR emission), we divide the observed TIR emission by a factor of 2 to be consistent with the model that only accounts for emission from the front side of the cloud. Because the [O I] line becomes optically thick relatively fast, we multiply the observed [O I] line emission by a factor of 2 to account for the clouds that have their optically thick side oriented towards us.

Based on the corrected line ratios $\left(\left[\mathrm{O}_{\mathrm{I}}\right] /\left[\mathrm{C}_{\mathrm{II}}\right]=0.61 \pm 0.15\right.$ and $\left.\left(\left[\mathrm{C}_{\mathrm{II}}\right]+\left[\mathrm{O}_{\mathrm{I}}\right]\right) / \mathrm{TIR}=0.047 \pm 0.009\right)$, PDR models predict an interstellar radiation field (ISRF) scaling factor, $G_{0}=31.6$, and a hydrogen gas density, $n_{\mathrm{H}}=10^{3.75} \mathrm{~cm}^{-3}$. The PDR line diagnostics suggest a stronger radiation field $\left(G_{0}=31.6\right)$ than derived from the dust spectral energy distribution (SED) modelling ( $G_{0}=1-3$, De Looze et al. 2016). It is plausible that geometry effects play an important role in the determination of the line ratios at the working resolution of a few tens of parsecs. With the [O I] line being detected merely in the very central $9.4 \operatorname{arcsec} \times 9.4 \operatorname{arcsec}$ (or $28 \times 28 \mathrm{pc}^{2}$ ) spaxel of the raster as opposed to the [C $\left.\mathrm{CI}\right]$ detection that covers an area of $110 \mathrm{pc} \times 75 \mathrm{pc}$, the actual [O $\mathrm{O}] /\left[\mathrm{C}_{\mathrm{II}}\right]$ line ratio (accounting for the source sizes) will be lower due to the [O I] emission not filling the entire beam. Accounting for the beam filling factors would shift the PDR model parameters $\left(G_{0}, n_{\mathrm{H}}\right)$ towards lower values, compatible with the SED fitting results on the spatial scales of $36 \operatorname{arcsec}$ or $\sim 100 \mathrm{pc}$. If we include the $\mathrm{CO}(1-0)$ line emission that was detected in the high-resolution BIMA CO map presented by Young (2001) in the PDR modelling (after correcting the $\mathrm{CO}(1-0)$ line emission by a factor of 2 to account for the optically thick CO clouds), we derive PDR model parameters of $G_{0}$ $=1.0$ and $n_{\mathrm{H}}=10^{4.25} \mathrm{~cm}^{-3}$ that are more consistent with the dust SED modelling results.

The origin of [C $\mathrm{CI}]$ and $\left[\mathrm{O}_{\mathrm{I}}\right]$ emission in NGC 185 might differ from the classical picture of collisional excitation in PDRs. Turbulent heating by shocks is likely to take place in NGC 185 based on the observation of shock-excited lines (e.g. $\mathrm{H}_{2} 0-0 \mathrm{~S} 0$ to $\mathrm{S}(6)$, [N II] $6584 \AA$, [S II] 6716, $6731 \AA$, [Fe II] $26 \mu \mathrm{m}$; Marleau et al. 2010; Martins et al. 2012). The importance of mechanical heating due to the turbulence in shocks has been shown to play an important role in PDRs (e.g. Appleton et al. 2013), even for the low-shock velocities (Lesaffre et al. 2013). The low warm-to-cold gas fraction in NGC 185 (see Section 4.3), however, suggests that the heating through shocks is negligible compared to radiative heating processes. Considering the old nature of the SNR in NGC 185 with an estimated shock velocity $\leq 85 \mathrm{~km} \mathrm{~s}^{-1}$ (Gonçalves et al. 2012), shock

\footnotetext{
${ }^{7}$ Although the metallicity of NGC 185 is sub-solar, we assume, to first order, that the effect of metallicity on the observed line ratios is negligible.
} 
excitation might be able to account for the observed [O I] emission in NGC 185 for shock velocities $\gtrsim 35 \mathrm{~km} \mathrm{~s}^{-1}$. The same model could provide at most 10 per cent of the observed [C $\mathrm{II}]$ emission in NGC 185. In case a significant fraction of the [O I] $63 \mu \mathrm{m}$ is excited by shocks, the PDR model parameters would shift to lower $G_{0}$ and $n_{\mathrm{H}}$. A possible contribution of the old stellar population to the TIR emission, on the other hand, would shift the data points to higher $G_{0}$ and $n_{\mathrm{H}}$ values.

Based on clear detections of the optical [O I] $6300 \AA$ A line (Martins et al. 2012) with a critical density $n_{\text {crit }}(\mathrm{H})=10^{6} \mathrm{~cm}^{-3}$ and the IR [Fe II] $26 \mu \mathrm{m}$ and [Si II] $34.8 \mu \mathrm{m}$ lines (Marleau et al. 2010) with critical densities of $n_{\text {crit }}(\mathrm{H})=2 \times 10^{6}$ and $3 \times 10^{5} \mathrm{~cm}^{-3}$, respectively, the presence of a denser $\left(n_{\mathrm{H}} \sim 10^{4-5} \mathrm{~cm}^{-3}\right)$ PDR region in the central regions of NGC 185 is also hinted at. We argue that the filling factor of these dense PDR regions is small compared to the rest of the gaseous ISM with the detection of the [O I] $63 \mu \mathrm{m}$ line limited to the central spaxel and based on the small cloud sizes measured from interferometric CO observations (Young \& Lo 1996). The PDR model parameters derived based on the $\mathrm{H}_{2} \mathrm{~S}(0), \mathrm{S}(1)$ and $\mathrm{S}(2)$ lines also suggest the presence of gas illuminated by radiation fields $G_{0} \geq 3 \times 10^{2}$ and gas densities $n_{\mathrm{H}} \sim 10^{4} \mathrm{~cm}^{-3}$. The detection of several tracers with high-excitation temperatures hints at the presence of a gas reservoir exposed to stronger radiation fields or would require alternative excitation mechanisms (e.g. shocks).

To derive a PDR surface temperature $(T \sim 40-70 \mathrm{~K})$ for NGC 185 , we rely on the average gas density $n_{\mathrm{H}} \sim 10^{3.75} \mathrm{~cm}^{-3}$ and a moderate radiation field $G_{0} \sim 1-10$ derived from dust SED fitting and PDR modelling. An average PDR temperature of $T \sim 50 \mathrm{~K}$ is used in Section 4.2 to obtain an upper limit for the molecular gas mass traced by $\left[\mathrm{C}_{\mathrm{I}}\right]$.

\section{TOTAL GAS RESERVOIR}

We combine the $\mathrm{H}$ I, $\mathrm{CO}(1-0)$, ionized gas, $\mathrm{H}_{2}$ and $\mathrm{X}$-ray observations to obtain total gas masses for NGC 147, NGC 185 and NGC 205. Table 3 provides an overview of the different gas mass measurements for the three galaxies.

\subsection{H I and CO observations}

For NGC 147, the $3 \sigma$ upper H I mass limit from Young \& Lo (1997) has been scaled to our adopted distance $\left(M_{\mathrm{H}_{\mathrm{I}}} \leq 3.7 \times 10^{3} \mathrm{M}_{\odot}\right)$. The $1 \sigma$ upper $\mathrm{CO}$ intensity limit $\left(I_{\mathrm{CO}} \leq 0.037 \mathrm{~K} \mathrm{~km} \mathrm{~s}^{-1}\right)$ reported by Sage et al. (1998) is used to derive a $3 \sigma$ upper limit $M_{\mathrm{H}_{2}} \leq 1.3$ $\times 10^{4} \mathrm{M}_{\odot}$ and $\leq 15.8 \times 10^{4} \mathrm{M}_{\odot}$ for a Galactic $\left(X_{\mathrm{CO}}=2.0 \times\right.$ $\left.10^{20} \mathrm{~cm}^{-2}\left[\mathrm{~K} \mathrm{~km} \mathrm{~s}^{-1}\right]^{-1}\right)$ and $H$-band luminosity-dependent $\left(X_{\mathrm{CO}}\right.$ $=24.3 \times 10^{20} \mathrm{~cm}^{-2}\left[\mathrm{~K} \mathrm{~km} \mathrm{~s}^{-1}\right]^{-1}$ ) conversion factor.

For NGC 185, we adopt the H I mass reported in Young \& Lo (1997), $M_{\mathrm{H}_{\mathrm{I}}}=1.1 \times 10^{5} \mathrm{M}_{\odot}$, scaled to our adopted distance. From the CO moment-0 map presented in Young (2001), we derive the $\mathrm{H}_{2}$ column density based on $\mathrm{N}_{\mathrm{H}_{2}}\left[\mathrm{~cm}^{-2}\right]=X_{\mathrm{CO}} I_{\mathrm{CO}(1-0)}$, with $I_{\mathrm{CO}(1-0)}$ the integrated line intensity in units of $\mathrm{K} \mathrm{km} \mathrm{s}^{-1}$. We apply two types of conversion factors: a Galactic conversion factor $\left(X_{\mathrm{CO}}=2.0 \times\right.$ $10^{20} \mathrm{~cm}^{-2}\left[\mathrm{~K} \mathrm{~km} \mathrm{~s}^{-1}\right]^{-1}$; Strong \& Mattox 1996; Dame et al. 2001; Ackermann et al. 2011) and a $H$-band luminosity-dependent conversion factor $\left(X_{\mathrm{CO}}=20.8 \times 10^{20} \mathrm{~cm}^{-2}\left[\mathrm{~K} \mathrm{~km} \mathrm{~s}^{-1}\right]^{-1}\right)$. Based on these two extremes, we find a molecular gas mass in the range $M_{\mathrm{H}_{2}}$ $=2.8-29.1 \times 10^{4} \mathrm{M}_{\odot}$.

In De Looze et al. (2012), we derived a total $\mathrm{H}$ I mass of $M_{\mathrm{H}_{\mathrm{I}}} \sim 4.0$ $\times 10^{5} \mathrm{M}_{\odot}$ for NGC 205 . The molecular gas mass $\left(M_{\mathrm{H}_{2}} \sim 2.1-13.1\right.$ $\left.\times 10^{5} \mathrm{M}_{\odot}\right)$ based on $\mathrm{CO}(1-0)$ observations of the northern and central regions in NGC 205 (Young \& Lo 1996; Welch et al. 1998) is recalculated for Galactic and $H$-band luminosity-dependent $X_{\mathrm{CO}}$ factors. We, furthermore, add the molecular gas mass derived from the new detections from our NRO 45 m observations $\left(M_{\mathrm{H}_{2}} \sim 0.2\right.$ $1.3 \times 10^{5} \mathrm{M}_{\odot}$, see Section 2.2), which sums up to a total molecular gas mass of $M_{\mathrm{H}_{2}} \sim 2.3-14.4 \times 10^{5} \mathrm{M}_{\odot}$.

\subsection{Cold CO-dark molecular gas}

Due to the multi-phase origin of the $\left[\mathrm{C}_{\mathrm{II}}\right]$ line emission (see Section 3.1), we would require a detailed ISM model to disentangle the $\left[\mathrm{C}_{\mathrm{II}}\right]$ emission that is originating from the molecular gas phase. Since such modelling requires an extensive set of multiphase tracers, we opt to probe the cold molecular gas mass that is not probed by $\mathrm{CO}$ observations based on $\left[\mathrm{C}_{\mathrm{I}}\right]$ line observations. The Herschel SPIRE FTS observations of NGC 205 allow us to derive $1 \sigma$ upper limits for the undetected $\left[\mathrm{C}_{\mathrm{I}}\right]$ line transitions (see Section 2.3). Based on those $1 \sigma$ upper limits for the $\left[\mathrm{C}_{\mathrm{I}}\right]$ line intensities, we derive upper limits on the molecular gas masses based on equation 12 in Papadopoulos, Thi \& Viti (2004):

$$
\begin{aligned}
M_{\mathrm{H}_{2}}= & 4.92 \times 10^{10} h^{\prime-2} \frac{(1+z-\sqrt{1+z})^{2}}{1+z}\left[\frac{X_{\mathrm{CI}}}{10^{-5}}\right]^{-1} \\
& \times\left[\frac{A_{\mathrm{ul}}}{10^{-7} \mathrm{~s}^{-1}}\right] Q_{\mathrm{ul}}^{-1}\left[\frac{I_{[\mathrm{CI}]}}{\mathrm{Jy} \mathrm{km} \mathrm{s}^{-1}}\right],
\end{aligned}
$$

where $h^{\prime}$ is defined as $H_{0}=100 h^{\prime}\left[\mathrm{km} \mathrm{s}^{-1} \mathrm{Mpc}^{-1}\right]$ with $H_{0}=$ $67.8 \mathrm{~km} \mathrm{~s}^{-1} \mathrm{Mpc}^{-1}$ (Planck Collaboration XIII 2016), $z$ is the redshift, $X_{\mathrm{CI}}$ is the neutral carbon abundance, $A_{\mathrm{ul}}$ is the Einstein coefficient for spontaneous emission, $Q_{\mathrm{ul}}$ is the excitation rate coefficient and $I_{[\mathrm{CI}]}$ is the $\left[\mathrm{C}_{\mathrm{I}}\right]$ line intensity. The Einstein coefficients $\left(A_{10}=\right.$ $\left.7.93 \times 10^{-8} \mathrm{~s}^{-1}, A_{21}=2.68 \times 10^{-7} \mathrm{~s}^{-1}, A_{20}=2 \times 10^{-14} \mathrm{~s}^{-1}\right)$ are taken from Papadopoulos et al. (2004). The $Q_{\mathrm{ul}}$ coefficients were calculated under non-local thermal equilibrium conditions for an average gas density $n_{\mathrm{H}} \sim 10^{3-4} \mathrm{~cm}^{-3}$ and gas temperature $T=50 \mathrm{~K}$ (see Section 3.3) following the recipes from Papadopoulos et al. (2004) and result in $Q_{10}=0.52$ and $Q_{21}=0.20$ for $n_{\mathrm{H}}=10^{3} \mathrm{~cm}^{-3}$, and $Q_{10}=0.45$ and $Q_{21}=0.31$ for $n_{\mathrm{H}}=10^{4} \mathrm{~cm}^{-3}$. The $\left[\mathrm{C}_{\mathrm{I}}\right] / \mathrm{H}_{2}$ abundance ratio is observed to range between the values of $10^{-5}$ and $10^{-4}$ in local and high-redshift galaxy samples (Frerking et al. 1989; Ikeda et al. 2002; Israel \& Baas 2003; Weiß et al. 2005; Walter et al. 2011). Simulations show that the neutral carbon abundance mostly varies with metallicity $\left(X_{[\mathrm{C} \mathrm{I}]} \propto \mathrm{Z}^{-1}\right)$ and hardly depends on radiation field strength, $G_{0}$ (Glover \& Clark 2016). We therefore assume a conservative lower limit $\left(\left[\mathrm{C}_{\mathrm{I}}\right] / \mathrm{H}_{2} \sim 10^{-5}\right)$ based on the low metallicity of the three dSphs.

Inserting those values in equation (4), we derive $1 \sigma$ upper molecular gas mass limits of $M_{\mathrm{H}_{2}} \leq 10^{5} \mathrm{M}_{\odot}$ for typical gas densities of $10^{3}$ and $10^{4} \mathrm{~cm}^{-3}$ in the CO peak in the north of NGC 205. Similar upper molecular gas mass limits of $M_{\mathrm{H}_{2}} \leq 10^{5} \mathrm{M}_{\odot}$ are derived in the centre of NGC 205 . We only provide the gas masses derived from the $\left[\mathrm{C}_{\mathrm{I}}\right] 1-0$ line transitions, since they give the tightest upper limits. These $1 \sigma$ upper mass limits are smaller than the molecular gas masses estimated from PDR models. For gas densities of $n_{\mathrm{H}} \sim$ $10^{3-4} \mathrm{~cm}^{-3}$ and a radiation field $G_{0} \in[1,10]$, the PDR models from Kaufman et al. (1999) predict line intensity ratios of [C II]/[C $\mathrm{I}] 1-0$ $\sim 1-10$ and $\left[\mathrm{C}_{\mathrm{I}}\right] 2-1 /\left[\mathrm{C}_{\mathrm{I}}\right] 1-0 \sim 2$ (see plots in the PDR toolbox; Pound \& Wolfire 2008). We derive a [C $\mathrm{II}] 158 \mu \mathrm{m}$ line flux of $1.4 \times 10^{-16} \mathrm{~W} \mathrm{~m}^{-2}$ (or $2212 \mathrm{Jy} \mathrm{km} \mathrm{s}^{-1}$ ) for NGC 205 from Herschel observations (De Looze et al. 2012). PDR models would thus predict line intensities of [C $\mathrm{C}] 1-0 \sim 221-2212 \mathrm{Jy} \mathrm{km} \mathrm{s}^{-1}$ and $\left[\mathrm{C}_{\mathrm{I}}\right]$ $2-1 \sim 110-1106 \mathrm{Jy} \mathrm{km} \mathrm{s}^{-1}$. Based on the PDR model predictions, we would expect both $\left[\mathrm{C}_{\mathrm{I}}\right]$ line transitions to be detected. The 
non-detection of the neutral carbon line transitions in NGC 205 might reflect a difference in filling factors between the ISM phases probed by $\left[\mathrm{C}_{\mathrm{II}}\right]$ and $\left[\mathrm{C}_{\mathrm{I}}\right]$. Studies of low-metallicity star-forming dwarf galaxies show that the filling factor of the cold dense molecular gas phase probed by $\left[\mathrm{C}_{\mathrm{I}}\right]$ is lower compared to the ISM phases probed by [C $\mathrm{II}]$ (De Looze et al., in preparation), which might also explain the non-detection of the $\left[\mathrm{C}_{\mathrm{I}}\right]$ lines in NGC 205. With the lowest $\mathrm{CO}$ transitions probing the cold and dense molecular gas, the filling factor of $\left[\mathrm{C}_{\mathrm{I}}\right]$-emitting clouds is thought to be more compatible with the $\mathrm{CO}$-emitting surfaces of dense clouds. Based on the integrated CO $1-0$ line intensity $\left(0.39 \mathrm{~K} \mathrm{~km} \mathrm{~s}^{-1}\right)$ observed with the NRAO $12 \mathrm{~m}$ telescope (FWHM $=55 \mathrm{arcsec}$ ) in the centre of NGC 205 (Welch et al. 1998) and the theoretical line ratio [C $\mathrm{I}]$ $1-0 /$ CO $1-0 \sim 25$ derived for a PDR model with $\left(G_{0}, n_{\mathrm{H}}\right)=(1$, $\left.10^{3.75} \mathrm{~cm}^{-3}\right)$, we can put a limit on the line intensity $I_{[\mathrm{CI}] 1-0} \sim$ $10^{-18} \mathrm{~W} \mathrm{~m}^{-2}$ in the centre of NGC 205, which is comparable to the $1 \sigma$ noise level in the SPIRE FTS spectra and consistent with the non-detection of [ $\left.\mathrm{C}_{\mathrm{I}}\right]$ in the smaller SPIRE FTS beam (FWHM $=40$ arcsec). Earlier works have already shown that the intensity and/or cloud filling factor of [ $\mathrm{C}_{\mathrm{I}}$ ] line emission predicted by PDR models can be inconsistent with observations and suggest a smaller filling factor for denser clouds (e.g. Keene et al. 1985; Schirm et al. 2014).

We can furthermore derive an upper limit on the CO-dark molecular gas reservoir based on the Herschel [C $\mathrm{CI}]$ observations of NGC 185. If we account for the negligible [C II] contribution from ionized gas and the estimated $[\mathrm{C} \mathrm{II}]$ emission from neutral atomic gas (see Section 3.1), we can invert equation (3) to derive the $\mathrm{C}^{+}$column density, $N_{\mathrm{C}+}$, in the molecular gas phase from the residual [C II] emission after subtracting the $\left[\mathrm{C}_{\mathrm{II}}\right]$ contribution from neutral atomic gas clouds. When adopting a critical density of $n_{\text {crit }}=7600 \mathrm{~cm}^{-3}$ for collisions with $\mathrm{H}_{2}$ molecules (see Goldsmith et al. 2012, derived for a kinetic gas temperature $T=20 \mathrm{~K}$ typical of molecular clouds) and assuming the best-fitting PDR model parameters $\left(n_{\mathrm{H}}=10^{3.75} \mathrm{~cm}^{-3}\right.$ and $T=50 \mathrm{~K}$, see Section 3.3), we derive a molecular gas mass of $\sim 4 \times 10^{4} \mathrm{M}_{\odot}$ for the [C II] emitting gas. Assuming a lower PDR gas density $\left(10^{3} \mathrm{~cm}^{-3}\right)$ could increase this molecular gas mass by a factor of $\sim 4$, the latter value should be regarded a strict upper limit since part of the $[\mathrm{C} \mathrm{II}]$ emission might originate from neutral atomic gas clouds rather than the molecular gas phase in PDRs. The latter upper limits on the CO-dark molecular gas reservoir are consistent with the upper limit of $\leq 10^{5} \mathrm{M}_{\odot}$ derived, based on the non-detection of [ $\left.\mathrm{C}_{\mathrm{I}}\right]$ line emission in NGC 205.

The [C $\mathrm{C}$ ]-based upper limits of the molecular gas mass $\left(M_{\mathrm{H}_{2}} \leq\right.$ $\left.1 \times 10^{5} \mathrm{M}_{\odot}\right)$ in NGC 205 are smaller compared to the $\mathrm{H}_{2}$ masses probed by $\mathrm{CO}\left(M_{\mathrm{H}_{2}}=2.8-29.1 \times 10^{5} \mathrm{M}_{\odot}\right)$. We thus conclude that the CO-dark molecular gas fraction is small in NGC 205 and that the CO line emission traces the bulk of molecular gas. Given that the ISM conditions are very similar in NGC 185, we believe that the latter argument can also be applied to NGC 185.

\subsection{Warm molecular gas}

The detection of rotational transitions of molecular hydrogen with the Spitzer IRS spectrometer (Marleau et al. 2010) suggests the presence of a reservoir of warm molecular gas in the centre of NGC 185 , significantly warmer than the PDR component traced by [C II] and [O I $]$. Based on the observed intensities of rotational $\mathrm{H}_{2}$ transitions for the three Spitzer IRS pointings in NGC 185 (see Fig. 1) reported by Marleau et al. (2010), we infer the temperature and column density of the warm molecular gas phase. Different mechanisms are capable of exciting $\mathrm{H}_{2}$ molecules, among which the radiative exci- tation by massive stars with photon energies $6 \leq \mathrm{h} v \leq 13.6 \mathrm{eV}$ in PDRs, and shock excitation in molecular outflows and supernova remnants are the most important contributors.

The detection of rotational $\mathrm{H}_{2}$ transitions up to $\mathrm{S}(7)$ (with excitation temperatures up to $5828 \mathrm{~K}$ ) seems unlikely to be driven by strong radiation (given the soft radiation field $G_{0} \sim 1-3$ derived from dust SED modelling; De Looze et al. 2016) and suggests that the highest $\mathrm{H}_{2}$ transitions are mainly shock-excited. We, therefore, restrict the fitting of representative temperatures and column densities to the lower rotational levels of $\mathrm{H}_{2}(\mathrm{~S}(0)$ to $\mathrm{S}(2))$ that are generally in collisional equilibrium (Burton, Hollenbach \& Tielens 1992).

Fig. 7 (top row) presents the excitation diagrams for the central, north and south region in NGC 185, respectively. Excitation diagrams visualize the distribution of different level populations described by the column density of the upper state $N_{\mathrm{u}}$ divided by the statistical weight $g_{\mathrm{u}}$ of that level population as a function of the upper state energy level $E_{\mathrm{u}} / k$. For the construction of this excitation diagram, we have assumed that optical depth effects are negligible, which should be appropriate given the low metal abundance of NGC 185.

The best fit to the $\mathrm{S}(0), \mathrm{S}(1)$ and $\mathrm{S}(2)$ lines results in a temperature $T \sim 180 \mathrm{~K}$ and column density $N_{\mathrm{H}_{2}} \sim 2.0 \times 10^{18} \mathrm{~cm}^{-2}$ in the central region of NGC 185 . Within the Spitzer IRS extraction area ( 35.7 arcsec $\times 10.7$ arcsec), the column density corresponds to a mass $M_{\mathrm{H}_{2}} \sim 111 \mathrm{M}_{\odot}$ of warm molecular gas. This warm molecular gas mass should be regarded as a lower limit, since the area covered by the IRS slit is limited. A similar analysis of the lower $\mathrm{H}_{2}$ transitions in the excitation diagram for the IRS slit positions observed in the north and south of the galaxy indicates warm molecular gas masses of $M_{\mathrm{H}_{2}} \sim 44 \mathrm{M}_{\odot}$ (for best-fitting parameters $T \sim 190 \mathrm{~K}$ and $\left.N_{\mathrm{H}_{2}} \sim 8.0 \times 10^{17} \mathrm{~cm}^{-2}\right)$ in the northern pointing and $M_{\mathrm{H}_{2}} \sim 87 \mathrm{M}_{\odot}$ (for best-fitting parameters $T \sim 190 \mathrm{~K}$ and $N_{\mathrm{H}_{2}} \sim 1.6 \times 10^{18} \mathrm{~cm}^{-2}$ ) towards the south of NGC 185 . The temperatures derived for the different regions in NGC 185 are lower compared to the average temperatures $(T \sim 350-380 \mathrm{~K})$ derived for low-metallicity star-forming dwarf galaxies in Cormier et al. (2014) due to the harder and stronger radiation fields in those star-forming dwarfs, but similar to the cold molecular gas temperatures $(T \sim$ $150 \mathrm{~K}$ ) derived for normal spiral galaxies (Roussel et al. 2007).

For the determination of the best-fitting temperature and column density from the observed excitation diagram, we assumed that the condition of local thermal equilibrium (LTE) is fulfilled for the lowest $\mathrm{H}_{2}$ transitions. Under LTE conditions, we expect to derive lower excitation temperatures for ratios of transitions with lower energy upper levels for an ortho-to-para density ratio of 3 (Burton et al. 1992) or, explicitly, $T(\mathrm{~S}(1)-\mathrm{S}(2))<T(\mathrm{~S}(1)-\mathrm{S}(3))<T(\mathrm{~S}(2)-$ $\mathrm{S}(3)$ ). Following the procedure in Roussel et al. (2007), we can determine the excitation temperature of consecutive transitions as a function of the ortho-to-para ratio (OPR) and, hereby, verify whether the diagram shows departures from thermalization of ortho-to-para levels. Fig. 7 (bottom panels) shows the determined excitation temperatures as a function of OPR for each pair of transitions from $\mathrm{S}(0)$ to $S(3)$, for the central, north and south IRS positions in NGC 185. The red coloured region satisfies the thermalization condition. The thermalization of $\mathrm{H}_{2}$ transitions up to $\mathrm{S}(3)$ seems satisfied only for the central region. Given that the $\mathrm{S}(3)-\mathrm{S}(2)$ ratio is not consistent with OPR $=3$ for the northern and southern regions, the higher rotational transition of $\mathrm{H}_{2}$ in these cases no longer satisfies collisional equilibrium and is likely excited by the shocks.

Given the violation of the LTE conditions in the north and south IRS positions of NGC 185, we might overestimate the excitation temperature (due to a shock contribution) and underestimate the 

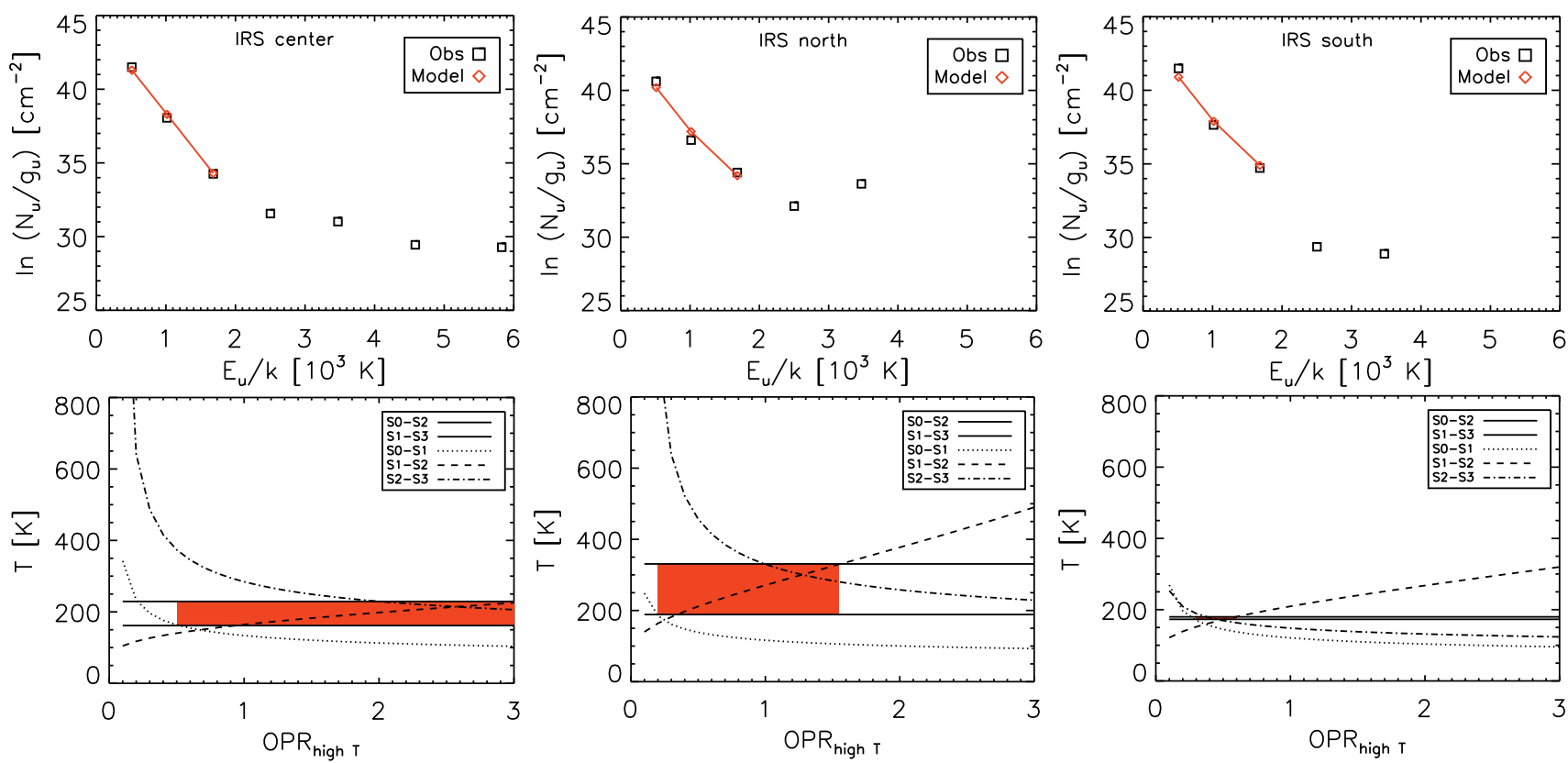

Figure 7. Top: observed $\mathrm{H}_{2}$ excitation diagrams in the central (left), north (middle) and south (right) IRS pointings for NGC 185 . The red line and symbols indicate the best-fitting model with excitation temperature, $T_{\mathrm{ex}}$, and column density, $N_{\mathrm{H}_{2}}$, fitted to the first three $\mathrm{H}_{2}$ rotational transitions $\mathrm{S}(0), \mathrm{S}(1)$, and $\mathrm{S}(2)$, for an OPR of 3. Bottom: diagram of the excitation temperatures derived for different pairs of rotational $\mathrm{H}_{2}$ transitions. The excitation temperatures should increase monotonically with higher order transitions to be compatible with the thermalization of $\mathrm{H}_{2}$ lines.

warm molecular gas mass in those regions. Even though the entire volume of warm molecular gas could not be traced due to the limited Spitzer IRS coverage, the observed warm-to-cold molecular gas fractions (ranging from 0.001 to 0.01 ) are one to two orders of magnitude lower than the typical warm-to-cold gas fractions reported by Roussel et al. (2007). We, therefore, do not expect to find massive reservoirs of warm molecular gas in NGC 185.

Due to the lack of IRS spectra for NGC 147 and NGC 205, we are not able to put constraints on the warm molecular gas reservoir in those galaxies.

\subsection{Ionized gas}

We estimate the ionized hydrogen mass from the observed $\mathrm{H} \alpha$ luminosity $\left(L_{\mathrm{H} \alpha} \sim 1.3 \times 10^{36} \mathrm{erg} \mathrm{s}^{-1}\right.$; Martínez-Delgado et al. 1999) in NGC 185 and convert it into an $\mathrm{H}_{\text {II }}$ mass (see equation 5 from Finkelman et al. 2010) assuming an electron temperature $T_{\mathrm{e}}=$ $10^{4} \mathrm{~K}$ and electron density $N_{\mathrm{e}} \sim 8300 \mathrm{~cm}^{-3}$ (which is the mean electron density determined for three PNe in NGC 185 by Gonçalves et al. 2012). The resulting $\mathrm{H}_{\mathrm{II}}$ mass $M_{\mathrm{H} \mathrm{II}}=1 \mathrm{M}_{\odot}$ is negligible compared to the neutral gas mass in NGC 185.

Similarly, the $1 \sigma$ noise level $\left(2.5 \times 10^{-17} \mathrm{erg} \mathrm{s}^{-1} \mathrm{~cm}^{-2} \operatorname{arcsec}^{-2}\right)$ in the $\mathrm{H} \alpha$ image of NGC 205 (Young \& Lo 1997) can be converted into a $3 \sigma$ upper mass limit $M_{\mathrm{H} \text { II }} \leq 1 \mathrm{M}_{\odot}$ assuming an electron temperature $T_{\mathrm{e}}=10^{4} \mathrm{~K}$ and electron density $N_{\mathrm{e}} \sim 5300 \mathrm{~cm}^{-3}$ (which is the mean electron density of PNe determined by Gonçalves et al. 2014).

We are not aware of any $\mathrm{H} \alpha$ observations for NGC 147 that would allow us to put a constraint on the ionized gas mass in this galaxy.

\subsection{Hot X-ray gas}

The non-detections of X-ray emission from NGC 147 and NGC 185 (Brandt et al. 1997) and NGC 205 (Welch et al. 1998) allow us to put a constraint on the reservoir of hot gas in these dSph galaxies. We calculate the upper X-ray gas mass limit based on the prescriptions from Roberts et al. (1991) and the X-ray and $B$-band ${ }^{8}$ luminosities of the galaxies. We find $1 \sigma$ upper limits on the X-ray gas mass of $\leq 0.2,0.2,3.8 \times 10^{4}$ for NGC 147, NGC 185 and NGC 205, respectively.

\subsection{Total gas mass}

For the computation of the total gas mass, we combine $\mathrm{H}$ I gas, $\mathrm{CO}$ traced and CO-dark molecular gas, ionized and hot X-ray gas masses and the warm molecular gas reservoir cooled by $\mathrm{H}_{2}$ rotational lines (see Table 3). The latter gas reservoirs combine to total gas masses of $M_{\mathrm{g}}=1.9 \times 10^{5}$ and $8.6 \times 10^{5} \mathrm{M}_{\odot}$ (corrected by a factor of 1.36 to account for helium) for NGC 185 and NGC 205, respectively, and an upper limit of $M_{\mathrm{g}} \leq 2.7 \times 10^{4} \mathrm{M}_{\odot}$ for NGC 147, assuming Galactic $X_{\mathrm{CO}}$ conversion factors. Using metallicity-dependent $X_{\mathrm{CO}}$ factors, the total gas masses are up to three times higher with gas masses of $M_{\mathrm{g}}=5.5 \times 10^{5}$ and $25.0 \times 10^{5} \mathrm{M}_{\odot}$ for NGC 185 and NGC 205 and an upper limit of $M_{\mathrm{g}} \leq 2.7 \times 10^{4} \mathrm{M}_{\odot}$ for NGC 147. If we assume Galactic $X_{\mathrm{CO}}$ factors, the gaseous reservoirs in NGC 185 and NGC 205 are dominated by the atomic H I gas component, while a metallicity-dependent $X_{\mathrm{CO}}$ factor would imply molecular gas reservoirs that are three to four times more massive than the atomic hydrogen content.

\section{STAR FORMATION EFFICIENCY}

Based on the gas mass measurements from Section 4, we can link the reservoir that is available for SF to the actual SFR, to learn more about the efficiency of gas consumption in $\mathrm{dSph}$ galaxies.

\footnotetext{
${ }^{8} B$-band luminosities are determined from the RC3 flux densities reported on NASA/IPAC Extragalactic Database (NED).
} 


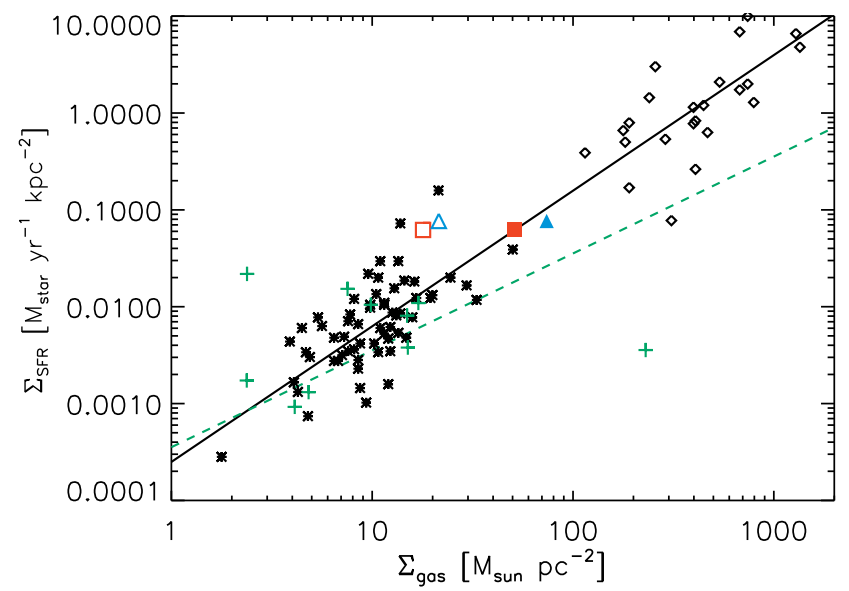

Figure 8. The Kennicutt-Schmidt relation between the gas surface density and the SFR surface density with a representative sample of spiral and starburst galaxies from Kennicutt (1998) indicated as black asterisks and diamonds, respectively. The locations of metal-poor star-forming dwarf galaxies studied by Cormier et al. (2014) are indicated with green crosses in the $\mathrm{K}-\mathrm{S}$ diagram (we omitted two galaxies with highly uncertain molecular gas masses). We only included the dwarfs from Cormier et al. (2014) with constraints on $\mathrm{H}_{\mathrm{I}}$ and $\mathrm{H}_{2}$ masses and scaling $\mathrm{CO}$ intensities with metallicitydependent $X_{\mathrm{CO}, Z}$ factors to obtain the latter masses. The dSph galaxies under analysis in this paper, NGC 185 and NGC 205, are marked as red squares and blue triangles. Empty symbols represent total gas masses $\left(\mathrm{H}+\mathrm{H}_{2}\right)$ with Galactic $X_{\mathrm{CO}}$ conversion factors, while filled symbols indicate total gas surface densities calculated with $H$-band luminosity-dependent $X_{\mathrm{CO}}$ factors. The black solid line represents the K-S relation $\Sigma_{\mathrm{SFR}}=\mathrm{A} \Sigma_{\text {gas }}^{N}$ with $N=1.4$ as found by Kennicutt (1998), where $\Sigma_{\text {gas }}$ is the total gas $\left(\mathrm{H}_{\mathrm{I}}+\mathrm{H}_{2}\right)$ surface density, while the green dashed line represents the average molecular gas depletion time-scale $\sim 2$ Gyr in a sample of nearby spiral galaxies derived by Leroy et al. (2013).

We derive total and molecular gas depletion time-scales $\tau_{\text {dep }}=$ $\Sigma_{\text {gas }} / \Sigma_{\text {SFR }}$, which is the time needed to exhaust the current total $\left(\mathrm{H} I+\mathrm{H}_{2}\right)$ and molecular $\left(\mathrm{H}_{2}\right)$ gas reservoir. Assuming that most of the gas content is located in the central regions $(150 \mathrm{pc} \times 90 \mathrm{pc})$ where recent $\mathrm{SF}\left(6.6 \times 10^{-4} \mathrm{M}_{\odot} \mathrm{yr}^{-1}\right)$ took place, we derive gas depletion time-scales of $\tau_{\mathrm{HI}+\mathrm{H}_{2}} \sim 0.3 \mathrm{Gyr}(0.8 \mathrm{Gyr})$ and $\tau_{\mathrm{H}_{2}} \sim$ $0.06 \mathrm{Gyr}(0.6 \mathrm{Gyr})$ for a Galactic ( $H$-band luminosity-dependent) $X_{\mathrm{CO}}$ factor for NGC 185 . Due to the lack of any recent SF activity and the non-detection of any gas in NGC 147, it is impossible to calculate a gas depletion time-scale for this galaxy.

We use constraints on the SFR $\sim 7 \times 10^{-4} \mathrm{M}_{\odot} \mathrm{yr}^{-1}$ (Monaco et al. 2009) in the central $28 \operatorname{arcsec} \times 26 \operatorname{arcsec}$ region of NGC 205. We calculate the total gas mass $\left(2.0 \times 10^{5} \mathrm{M}_{\odot}\right)$ in this central region of NGC 205 based on the atomic hydrogen mass $\left(M_{\mathrm{HI}} \sim\right.$ $7.6 \times 10^{4} \mathrm{M}_{\odot}$ ) of two central Hi clumps (Young \& Lo 1997) and the molecular gas mass $\left(M_{\mathrm{H}_{2}} \sim 6.8 \times 10^{4} \mathrm{M}_{\odot}\right.$, assuming a Galactic $X_{\mathrm{CO}}$ factor) from the central CO pointing of Welch et al. (1998), scaled by a factor of 1.36 to include helium. Using a $H$-band luminosity-dependent $X_{\mathrm{CO}}$ factor $\left(12.5 \times 10^{20} \mathrm{~cm}^{-2}\left[\mathrm{~K} \mathrm{~km} \mathrm{~s}^{-1}\right]^{-1}\right)$ would imply a molecular gas reservoir of $4.2 \times 10^{5} \mathrm{M}_{\odot}$. We derive gas depletion time-scales of $\tau_{\mathrm{HI}+\mathrm{H}_{2}} \sim 0.3 \mathrm{Gyr}(1.0 \mathrm{Gyr})$ and $\tau_{\mathrm{H}_{2}} \sim 0.1 \mathrm{Gyr}(0.8 \mathrm{Gyr})$ for a Galactic ( $H$-band luminositydependent) $X_{\mathrm{CO}}$ factor for NGC 205.

Based on these gas mass depletion time-scales, the $\mathrm{dSph}$ galaxies, NGC 185 and NGC 205, are forming stars more actively in comparison to galaxies on the Kennicutt (1998) relation (see Fig. 8). At the current depletion time-scale, the entire molecular gas reservoir (based on a Galactic $X_{\mathrm{CO}}$ factor) would be exhausted within less than 100 Myr. For metallicity-dependent $X_{\mathrm{CO}}$ factors, the star formation efficiencies are more comparable to normal spiral galaxies, but molecular gas depletion time-scales still about a factor of 2 lower compared to the average $\Sigma_{\mathrm{H}_{2}} \sim 2 \mathrm{Gyr}$ in a sample of nearby spiral galaxies derived by Leroy et al. (2013). Although the level of $\mathrm{SF}$ in NGC $185\left(\mathrm{SFR} \sim 6.6 \times 10^{-4} \mathrm{M}_{\odot}\right)$ and NGC $205(\mathrm{SFR} \sim$ $\left.7 \times 10^{-4} \mathrm{M}_{\odot}\right)$ is very different from the SFRs observed in normal star-forming galaxies $\left(1-10 \mathrm{M}_{\odot} \mathrm{yr}^{-1}\right)$, the SFR conditions in the centres of those dSphs do seem to approach the star-forming conditions observed in local spirals (and might be even more efficient). Independent evidence for a high star formation efficiency (SFE) in NGC 185 was derived from its observed abundance [O/Fe] ratio (0.8 dex; Gonçalves et al. 2012), which indicates a higher SNe II rate compared to $\mathrm{SNe}$ Ia. The positive $[\mathrm{O} / \mathrm{Fe}]$ ratio suggests that most of the gas has been consumed on short time-scales.

Alternatively, we might be observing these two dSph galaxies towards the end of their recent SF episode, having already burned most of their initial gas reservoir and resulting in an artificially high SFE estimate. We also caution that the SFE predictions might be affected by stochastic effects due to the small number of clouds detected within these galaxies.

\section{GAS-TO-DUST MASS RATIO}

Combining all gas and dust mass measurements (see Table 3), we derive estimates of the global gas-to-dust mass ratios GDR $\sim 37$ in NGC 185 and GDR $~ 48$ in NGC 205. Using metallicity-dependent $X_{\mathrm{CO}}$ factors, the global gas-to-dust mass ratio would increase to GDR $\sim 107$ for NGC 185 and GDR $\sim 139$ for NGC 205. Since the $\mathrm{HI}$ gas is more extended compared to the dust in NGC 185 and NGC 205 (see Figs 2 and 4, respectively), the gas-to-dust mass ratio might become even smaller on local scales. These global values are lower than the average Galactic gas-to-dust mass ratio $\sim 130$ (Draine \& Li 2007). Based on the observed trend of increased gasto-dust mass ratios with decreasing metallicity (e.g. Lisenfeld \& Ferrara 1998; James et al. 2002; Hunt, Bianchi \& Maiolino 2005; Engelbracht et al. 2008; Galliano, Dwek \& Chanial 2008; Galametz et al. 2011; Magrini et al. 2011; Rémy-Ruyer et al. 2014), these low gas-to-dust mass ratios are considered even more exceptional, where a simple GDR $\propto Z^{-1}$ scaling would imply a GDR $\sim 370$ for NGC 185 and GDR 520 for NGC 205.

Similarly low gas-to-dust mass ratios were observed in the elliptical galaxy, NGC 4125 (Wilson et al. 2013), and the dust-lane lenticular galaxy, NGC 5485 (Baes et al. 2014). The low gas-to-dust mass ratio in NGC 4125 was attributed to the rapid heating of gas to temperatures $\gtrsim 10^{4} \mathrm{~K}$, faster than the evaporation of cold dust in this galaxy (Wilson et al. 2013). Such a scenario seems, however, unlikely for NGC 185, where the warm-to-cold molecular gas fractions (0.001-0.01) are much lower than observed in more massive star-forming galaxies. Also, the non-detection of X-ray emission (see Section 4.5) in NGC 185 and NGC 205 is able to put an upper limit on the reservoir of hot gas $\left(\lesssim 0.2-3.8 \times 10^{4} \mathrm{M}_{\odot}\right)$.

None of the chemical evolution models, including interstellar grain growth (Asano et al. 2013) and accounting for a wide variation of SPH (Zhukovska 2014) (see figs 8 and 9 in Rémy-Ruyer et al. 2014), predict a gas-to-dust ratio as low as that observed in these dSphs considering its metal abundance. Since these low GDRs clearly deviate from theoretical model predictions, it is worth investigating the origin of the discrepancy between model and observations.

First, we consider possible caveats in the determination of total gas and dust masses. Given that the $\mathrm{HI}$ and $\mathrm{CO}$ observations 

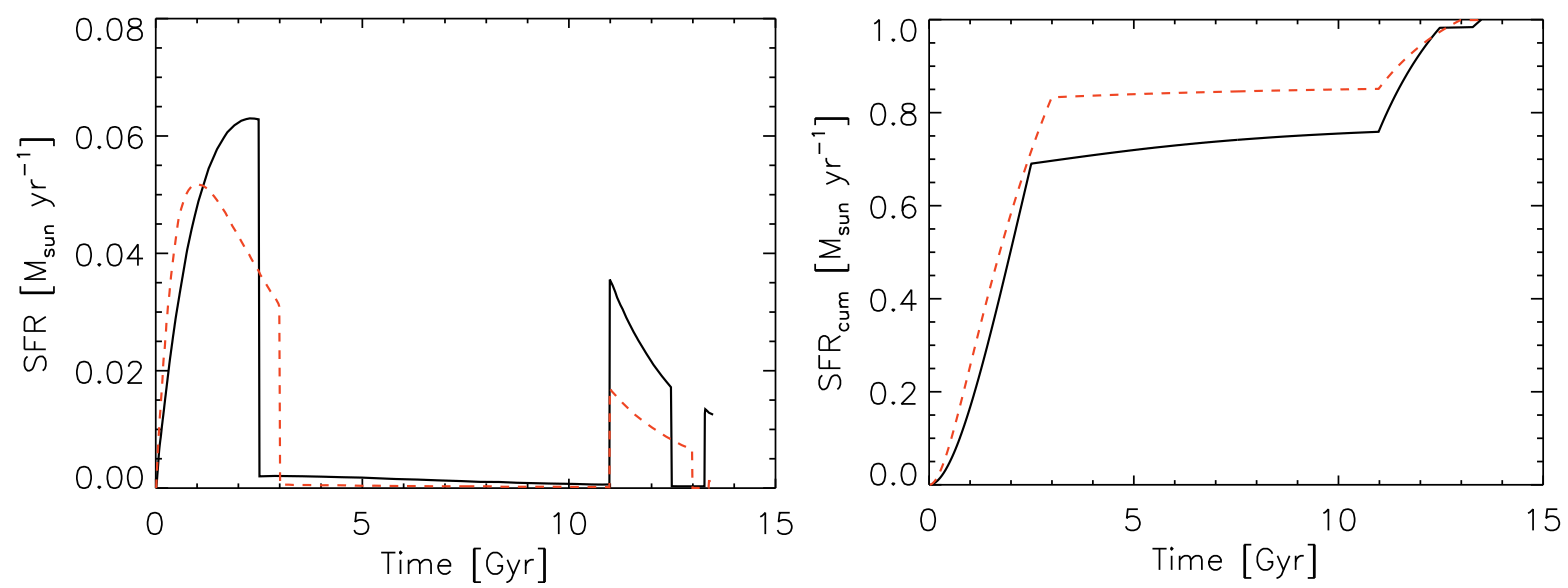

Figure 9. Left: the two SFHs for NGC 185 used in Martins et al. (2012) (left-hand panel) and their corresponding cumulative SFH (right-hand panel).

are sufficiently deep to detect faint emission (Young \& Lo 1997; Young 2001), we are confident that the current $\mathrm{H}$ I and CO data sets will not miss a massive reservoir of atomic or molecular gas. The warm molecular gas masses might be underestimated due to model assumptions and/or insufficient observational coverage. It is, however, unrealistic to assume that the $\mathrm{H}_{2}$ observations with Spitzer can account for a substantial massive gas reservoir given the low warmto-cold molecular ratio (see Section 4.3). The presence of a massive ionized gas reservoir is also unlikely given the weak $\mathrm{H} \alpha$ emission from NGC 185. The non-detection of [ $\mathrm{C}_{\mathrm{I}}$ ] in NGC 205 implies that the CO-dark molecular gas content is insignificant compared to the $\mathrm{H}_{2}$ mass traced by $\mathrm{CO}$.

Although the dust masses in NGC 185 and NGC 205 have been robustly measured in De Looze et al. $(2016,2012)$, the lack of knowledge on the dust composition and dust mass absorption coefficients makes the derived dust masses uncertain by at least a factor of 2. Even with this uncertainty factor of 2, the main cause for the low gas-to-dust mass ratios seems hard to explain based on a lack of observational constraints and/or inaccuracies in the ISM mass predictions. We attribute the low gas-to-dust mass ratios to a combination of possible effects including efficient dust production and long-term grain survival (see De Looze et al. 2016 and Section 7.1) and the removal of part of the gas mass from the galaxy (see Section 7.2).

\section{THE ISM MASS BUDGET}

In this section, we discuss the origin of gas and dust reservoirs in NGC 147, NGC 185 and NGC 205. Hereto, we compare the gaseous reservoirs detected in these galaxies to theoretical predictions from a simple closed-box model.

\subsection{Theoretical gas consumption and replenishment}

Based on the prescriptions of Faber \& Gallagher (1976), Sage et al. (1998) and Welch et al. (1998) estimated the gas mass returned to the ISM by PNe in the three dSph galaxies NGC $147\left(6-11 \times 10^{5} \mathrm{M}_{\odot}\right)$, NGC $185\left(8-17 \times 10^{5} \mathrm{M}_{\odot}\right)$ and NGC $205\left(23 \times 10^{5} \mathrm{M}_{\odot}\right)$, which are similar to or in excess of the current gas content in those galaxies. As a proof of concept, we redo these calculations for the $\mathrm{dSph}$
NGC $185^{9}$ based on a simple chemical evolution model with a closed-box approximation to account for the gas and dust mass returned by $\mathrm{PNe}$ and supernovae. In this simple model, we calculate the gas and dust mass that has been returned by the intermediateage population (2-3 Gyr) based on the best-fitting SFH presented by Martins et al. (2012) that were optimized to fit the abundance ratios of $\mathrm{PNe}$, the age-metallicity relation and the total galaxy mass at the present day. Fig. 9 shows their two best-fitting SFHs (lefthand panel) and the cumulative SFHs (right-hand panel). The latter corresponds well to the cumulative SFH presented by Geha et al. (2015) that was derived from colour-magnitude diagrams based on deep $V$ - and I-band Hubble Space Telescope ACS observations. Also, the average current SFR 2.9-3.8 $\times 10^{-3} \mathrm{M}_{\odot} \mathrm{yr}^{-1}$ measured over a time period of $1 \mathrm{Gyr}$, is consistent with the SFR derived by Martínez-Delgado et al. (1999) (SFR $~ 6.6 \times 10^{-4} \mathrm{M}_{\odot} \mathrm{yr}^{-1}$ ), considering that the latter value only accounts for SF that took place in the central regions of NGC 185.

During the simulation, we track the gas consumption, dust production and return of gaseous material to the ISM based on these SFHs at individual timesteps of $10 \mathrm{Myr}$. At every timestep, the contribution from stars with lifetimes $\tau_{m}=t-t_{0}$ (with $t_{0}$ the age at which the galaxy was born) is taken into account. The stellar lifetimes for stars of a different mass and metallicity are calculated based on the parametrization of Raiteri, Villata \& Navarro (1996). The dust yields are taken from Zhukovska, Gail \& Trieloff (2008) and Bianchi \& Schneider (2007) for intermediate- $\left(0 \mathrm{M}_{\odot}<M<\right.$ $\left.8 \mathrm{M}_{\odot}\right)$ and high-mass $\left(12 \mathrm{M}_{\odot}<M<40 \mathrm{M}_{\odot}\right)$ stars, respectively. The gas yields from van den Hoek \& Groenewegen (1997) and Woosley \& Weaver (1995) are used for intermediate-mass and massive stars, respectively. We interpolate between the intermediateand high-mass estimates to derive dust and metal yields for stars with masses $8 \mathrm{M}_{\odot}<M<12 \mathrm{M}_{\odot}$. We neglect stars more massive than $40 \mathrm{M}_{\odot}$ since they will collapse to form black holes at the end of their lives and have a negligible contribution to the enrichment of the ISM. For our calculations, we assume a Salpeter (1955) initial mass function with a slope of -2.35 within a mass range from 0.1 to $100 \mathrm{M}_{\odot}$.

Running the simulation with an initial gas mass of $M_{\text {gas }}(t=0)$ $=10^{7.5} \mathrm{M}_{\odot}$ and metal abundance $[\mathrm{O} / \mathrm{H}](t=0) \sim 10^{-4}$ (consistent with the initial conditions used by Martins et al. 2012), we derive

\footnotetext{
${ }^{9}$ We refrain from redoing the calculations for NGC 147 and NGC 205 due to the lack of sufficient constraints on their recent SFH.
} 
a gas mass $\left(3-6 \times 10^{5} \mathrm{M}_{\odot}\right)$ returned to the ISM since the second burst of SF initiated $\sim 3.5$ Gyr ago until the current epoch. The total gas reservoir at the current epoch is estimated to be $1.1 \times 10^{6} \mathrm{M}_{\odot}$. With an observed gas mass $\left(1.9-5.5 \times 10^{5} \mathrm{M}_{\odot}\right.$, see Section 4) that is two to five times smaller than predicted by our simple closed box model (and up to three times smaller than the gas mass returned to the ISM during the last two SF episodes), we argue that the closed box approximation does not fit the observational constraints. Similarly, De Looze et al. (2012) showed that the current gas mass reservoir in NGC 205 is too low compared to the predictions of the gas mass returned by $\mathrm{PNe}$. The non-detection of a gaseous reservoir in NGC 147 furthermore seems unlikely, given the population of evolved stars in this galaxy (Davidge 2005). Based on a comparison of the observed dust and gas reservoirs in NGC 185 with a simple closed box model, we argue the low gas-to-dust mass ratio and gas deficiency result from gas removal processes. This gas removal could be induced by internal mechanisms (e.g. supernova explosions, stellar winds) and/or tidal interactions (see Section 7.2).

In De Looze et al. (2016), we showed that the observed dust content is higher compared to the dust mass produced by AGB stars and supernovae during the last $100 \mathrm{Myr}$ (which is the estimated dust survival time) in NGC 185. Based on the closed box model presented in this paper, we predict the production of up to $400 \mathrm{M}_{\odot}$ during the last 100 Myr. To account for the observed dust mass in NGC 185 $\left(5.1 \times 10^{3} \mathrm{M}_{\odot}\right)$, we would require an efficient dust production during the last $1.6 \mathrm{Gyr}$ without any grain destruction. The latter dust survival time is significantly higher than the estimated dust lifetime in NGC 185 (50-100 Myr). In De Looze et al. (2016), we had argued that grain growth in the dense ISM phases could be an additional source of dust production, but the mechanisms that would enable the accretion of material on to grain surfaces in the ISM are not well understood (Ferrara, Viti \& Ceccarelli 2016). Other than longer dust survival times, the metal production in current nucleosynthesis models and the dust yields of AGB stars and supernovae in dust nucleation models might be underestimated.

\subsection{Gas removal}

Gas removal can result from internal mechanisms (e.g. supernova explosions, stellar winds) or external influences (e.g. hydrodynamical or gravitational interactions). Based on analytic/numerical models for dark matter haloes with the inclusion of stellar feedback (Ferrara \& Tolstoy 2000), a total blow-away of the entire gaseous medium is only possible for dark matter haloes of $M_{\mathrm{g}} \sim 5 \times 10^{6}$ $\mathrm{M}_{\odot}$ (Ferrara \& Tolstoy 2000). With galaxy masses of $M_{\mathrm{g}} \sim 7.2 \times$ $10^{8} \mathrm{M}_{\odot}$ and $5.6 \times 10^{8} \mathrm{M}_{\odot}$ (Geha et al. 2010) for NGC 185 and NGC 147, the dSph galaxies might lose some (but not all) gas to the intergalactic medium. The latter scenario is consistent with chemical evolution models that require efficient galactic winds to reproduce observed gas masses and abundances for the dSph galaxy population (e.g. Lanfranchi \& Matteucci 2004, 2010; Martins et al. 2012). Some of the metal-enriched gas expelled by galactic winds is assumed to rain back down on the galaxy disc according to a 'galactic fountain' mechanism that is able to flatten the metallicity gradient in those dSphs (De Young \& Heckman 1994; Ferrara \& Tolstoy 2000; Barazza \& Binggeli 2002).

While this 'galactic fountain' effect might work for heavier galaxies, we argue that any gas expelled from these Andromeda dSph dwarfs will easily escape from the galaxy if heated to sufficiently high temperatures. Using the total galaxy masses (including baryonic and dark matter) for NGC 147, NGC 185 and NGC 205 from
De Rijcke et al. (2006) within two effective radii $\left(R_{\text {eff }}\right)$, we derive escape velocities ${ }^{10}$ that range from 57 to $91 \mathrm{~km} \mathrm{~s}^{-1}$ at a radius of $2 * R_{\text {eff }}$. By heating the gas to sufficiently high temperatures, the thermal gas velocity of a gas with a temperature of $T_{\text {kin }}=10^{6} \mathrm{~K}$ $\left(v_{\text {th }}=90 \mathrm{~km} \mathrm{~s}^{-1}\right)^{11}$ would be sufficient for the gas no longer to be gravitationally bound to the galaxy. This simple calculation shows that if the hot gas is blown out by supernova feedback and/or stellar winds to large radii, it might be able to escape from the galaxy if heated to sufficiently high temperatures. The typical hot X-ray halo of gas that provides more massive galaxies with fresh gas supplies for SF does not seem to be present in those lower metallicity dwarfs, which is observationally supported by the non-detections of X-ray emission in these dwarfs (see Section 4.5). The presence of dust and the metal enrichment $(0.2-0.3 \mathrm{dex})$ over the last $\sim 8 \mathrm{Gyr}$ (Gonçalves et al. 2012) in the central regions of NGC 185 is consistent with the absence of 'galactic fountains' that would distribute the metals throughout the galaxy's disc.

But this observed central concentration of metals is also compatible with a scenario of external influences that mostly remove the metal-poor H I gas from the outer galaxy parts (Valluri \& Jog 1990). Tidal interactions with other satellite galaxies or Andromeda can also potentially remove the gas from the outer galaxy regions in NGC 185 and entirely strip the gas from NGC 147. Recent observations from the Pan-Andromeda Archaeological Survey reveal isophotal twisting and the emergence of extended tidal tails in NGC 147, but do not show any evidence for tidal effects on the stellar light profiles of NGC 185 (Crnojević et al. 2014). The asymmetric $\mathrm{H}_{\mathrm{I}}$ distribution, combined with its small extent up to only one-fourth of its Holmberg radius (Young \& Lo 1997) might be an indication for tidal effects having played an important role in the evolution of NGC 185 in the past. Being located at an angular distance of $12^{\circ}$ from M 31, NGC 185 and NGC 147 are currently beyond the tidal influence radius of Andromeda. ${ }^{12}$ Given their small angular separation $\left(\sim 1^{\circ}\right)$, the two galaxies have been argued to form a gravitationally bound pair (van den Bergh 1998; Geha et al. 2010). Based on their carbon star populations (Battinelli \& Demers 2004), the different ages of the dominant old stellar populations (suggesting their separate infall into the group system, Geha et al. 2015), timing arguments (Watkins, Evans \& van de Ven 2013) and conditions for their gravitational bound (Evslin 2014), there is however no reason to assume that the two dSphs form a close pair. Watkins et al. (2013) rather suggest a close connection between NGC 185 and Cass II. The possible bound with the dSph galaxy Cass II might have had an influence on the recent SF in NGC 185. Earlier interactions with Andromeda (or even the Milky Way; Teyssier, Johnston \& Kuhlen 2012) could be responsible for significant gas removal in the past. It is, for the moment, unclear whether tidal interactions with M 31 or mutual encounters between the dwarf satellites (NGC 147, NGC 185, Cass II) have caused the gas stripping in the two galaxies. In NGC 205, there is observational evidence for a past tidal interaction with the Andromeda galaxy that could have removed part of the gas content of NGC 205 (see De Looze et al. 2012 for more details). Better knowledge on the orbits of the three dSph galaxies

\footnotetext{
${ }^{10}$ The escape velocity is calculated from $v_{\mathrm{esc}}=\sqrt{2 G M / R}$ using the total galaxy mass, $M$, the distance to the centre of mass, $R=2 * R_{\text {eff }}$, and the gravitational constant, $G$.

${ }^{11}$ The thermal gas velocity for a gas with kinetic temperature $T_{\text {kin }}=10^{6} \mathrm{~K}$ is calculated from $v_{\text {th }}=\sqrt{k_{\mathrm{B}} * T / m}$ using the Boltzmann constant, $k_{\mathrm{B}}$, the mass of a hydrogen atom, $m$, and the gas temperature, $T_{\text {kin }}=10^{6} \mathrm{~K}$.

${ }^{12}$ For NGC 185 and NGC 147, the tidal radius has been calculated to be between 10 and $12 \mathrm{kpc}$ or, thus, beyond 25 effective radii (Geha et al. 2010).
} 
is required to model the past interactions with companions in the Andromeda group.

\subsection{Galaxy evolution}

Although the three dSph galaxies probably share a similar evolutionary history (driven by galactic winds and/or tidal interactions), their different ISM conditions (i.e. central ongoing SF in NGC 185 and NGC 205 and the lack of any detectable ISM material in NGC 147) shows that similar mechanisms can result in a variety of morphological outcomes (e.g. Ryś, Falcón-Barroso \& van de Ven 2013), depending on the efficiency of galactic winds and the orbit of the galaxy (e.g. Kazantzidis et al. 2011).

Chemical evolution models seem to require high galactic wind efficiencies to explain the build-up of gas and metals in these galaxies (e.g. Lanfranchi \& Matteucci 2004, 2010; Martins et al. 2012), while tidal stirring (Mayer et al. 2001) and galaxy threshing (Bekki, Couch \& Drinkwater 2001) have been put forward as the most important mechanisms for the formation of dSphs and ultracompact dwarfs in low-density group environments based on the galaxy simulations. Also, observational evidence of tidal influence for the galaxies residing in group environments (e.g. Paudel \& Ree 2014) supports these theoretical simulations.

Given the wide range of resulting end products and the continuous influence of environmental effects on most galaxies residing in cluster and group environments, it is hard to constrain the progenitor galaxies of these $\mathrm{dSph}$ galaxies in the Local Group (Lisker et al. 2013). Rather than the transformation of dwarf irregular into dwarf elliptical galaxies, the present-day dwarf galaxy population might originate from the same common progenitor population that experienced a different evolution due to differences in dark matter content, stellar mass and/or environments (e.g. Ferrara \& Tolstoy 2000; Tolstoy et al. 2009; Sawala, Scannapieco \& White 2012). The only way to properly constrain the evolutionary history of the population of dwarf spheroidal galaxies, i.e. present day-observed in group and cluster environments, is through a combination of observations probing their stellar populations, SFH, chemical enrichment, kinematic properties (e.g. Tolstoy et al. 2009) and orbital parameters (e.g. Howley et al. 2008; Watkins et al. 2013).

\section{CONCLUSIONS}

We make an inventory of the gas content in three low-metallicity dSph galaxies of the Local Group (NGC 147, NGC 185 and NGC 205) based on an extensive set of ancillary observations. We present new Nobeyama $\mathrm{CO}(1-0)$ observations that cover the previously unexplored regions in the south of NGC 205 and we use Herschel SPIRE FTS [C $\mathrm{C}_{\mathrm{I}}$ ] observations to limit the fraction of CO-dark gas in NGC 205. Based on Herschel observations of the far-IR fine-structure lines $[\mathrm{C} \mathrm{II}]$ and $\left[\mathrm{O}_{\mathrm{I}}\right]$ towards the central regions NGC 185, we analyse the typical conditions of the ISM in dSphs.

We compute total gas masses of $M_{\mathrm{g}}=1.9-5.5 \times 10^{5} \mathrm{M}_{\odot}$ (NGC 185) and 8.6-25.0 × 105 $\mathrm{M}_{\odot}$ (NGC 205) within the limits of uncertainty on the $X_{\mathrm{CO}}$ factors, by combining the mass reservoirs of atomic, cold and warm molecular, ionized and hot X-ray gas. Non-detections result in an upper gas mass limit of $M_{\mathrm{g}} \leq 0.3-2.2 \times$ $10^{5} \mathrm{M}_{\odot}$ for NGC 147. Our new NRO $45 \mathrm{~m} \mathrm{CO}(1-0)$ map of the southern regions in NGC 205 shows that most of the molecular gas is distributed towards the north and centre. The non-detections of the [C I ] 1-0 and 2-1 line transitions in the SPIRE FTS spectra imply that the CO-dark gas fraction is negligible in NGC 205 compared to the molecular gas mass traced by $\mathrm{CO}$, which is also consistent with the lower $\left[\mathrm{C}\right.$ II]/CO ratios $\left(2-4 \times 10^{3}\right)$ in $\mathrm{dSphs}$ compared to low-metallicity star-forming dwarf galaxies with $\left[\mathrm{C}_{\mathrm{II}}\right] / \mathrm{CO}$ ratios of a few times $10^{4}$.

Photodissociation models suggest a soft radiation field $\left(G_{0} \sim 1-\right.$ 30 ) and moderate hydrogen gas density $\left(n_{\mathrm{H}} \sim 10^{3.75}-10^{4.25} \mathrm{~cm}^{-3}\right)$ to explain the observed [C $\mathrm{CI}],\left[\mathrm{O}_{\mathrm{I}}\right]$ and total-IR emission in NGC 185. The detection of several high-excitation lines implies that also a dense PDR phase with small filling factor is present or alternatively requires shocks to excite the lines. The high $[\mathrm{C}$ II]/TIR $\sim 1.5$ per cent and $\left[\mathrm{C}_{\mathrm{II}}\right]+\left[\mathrm{O}_{\mathrm{I}}\right] / \mathrm{TIR} \sim 2$ percent ratios indicate that the photoelectric efficiency is high, which might be explained by a high PAH abundance and/or low level of grain charging in NGC 185. The SFR densities and current gas reservoirs in NGC 185 and NGC 205 places these galaxies above the main sequence of starforming galaxies. The short molecular gas depletion time-scales imply that fuel for SF will run out in less than a few $100 \mathrm{Myr}$ in these dSphs.

We derive global gas-to-dust mass ratios of GDR 37-107 and 48-139 that are at the low end of the average Milky Way ratio of GDR $\sim 130$ and significantly lower compared to the expected ratios of GDR $\sim 370$ and 520 for the metal abundances in NGC 185 $\left(0.36 \mathrm{Z}_{\odot}\right)$ and $\mathrm{NGC} 205\left(0.25 \mathrm{Z}_{\odot}\right)$, respectively. Based on a simple closed box model, we confirm that these $\mathrm{dSphs}$ are gas-deficient and the dust has a longer dust survival time $(\sim 1.6 \mathrm{Gyr})$ in these galaxies, which can also explain their anomalous GDR. We conclude that part of the gas content has been removed from the $\mathrm{dSph}$ satellites in the recent past. We believe that efficient galactic winds (combined with the heating of gas to sufficiently high temperatures in order for it to escape from the galaxy) and/or environmental interactions with neighbouring galaxies are responsible for the gas removal from NGC 147, NGC 185 and NGC 205.

\section{ACKNOWLEDGEMENTS}

The authors would like to thank Marla Geha and Martha Boyer for interesting discussions that have helped to improve this paper. We would like to thank Denise Gonçalves and Laura Magrini for kindly sharing their $\mathrm{H} \alpha$ data presented in Gonçalves et al. (2012). IDL gratefully acknowledges the support of the Science and Technology Facilities Council (STFC) and the Flemish Fund for Scientific Research (FWO-Vlaanderen). PACS has been developed by a consortium of institutes led by MPE (Germany) and including UVIE (Austria); KU Leuven, CSL, IMEC (Belgium); CEA, LAM (France); MPIA (Germany); INAFIFSI/ OAA/OAP/OAT, LENS, SISSA (Italy); IAC (Spain). This development has been supported by the funding agencies BMVIT (Austria), ESA-PRODEX (Belgium), CEA/CNES (France), DLR (Germany), ASI/INAF (Italy), and CICYT/ MCYT (Spain). SPIRE has been developed by a consortium of institutes led by Cardiff University (UK) and including Univ. Lethbridge (Canada); NAOC (China); CEA, LAM (France); IFSI, Univ. Padua (Italy); IAC (Spain); Stockholm Observatory (Sweden); Imperial College London, RAL, UCLMSSL, UKATC, Univ. Sussex (UK); and Caltech, JPL, NHSC, Univ. Colorado (USA). This development has been supported by national funding agencies: CSA (Canada); NAOC (China); CEA, CNES, CNRS (France); ASI (Italy); MCINN (Spain); SNSB (Sweden); STFC and UKSA (UK); and NASA (USA). This research has made use of the NED that is operated by the Jet Propulsion Laboratory, California Institute of Technology, under contract with the National Aeronautics and Space Administration. 


\section{REFERENCES}

Ackermann M. et al., 2011, ApJ, 726, 81

Aniano G., Draine B. T., Gordon K. D., Sandstrom K., 2011, PASP, 123, 1218

Appleton P. N. et al., 2013, ApJ, 777, 66

Asano R. S., Takeuchi T. T., Hirashita H., Inoue A. K., 2013, Earth Planets Space, 65, 213

Asplund M., Grevesse N., Sauval A. J., Scott P., 2009, AR\&A, 47, 481

Baade, W.1951, Publ. Michigan Obs., 10, 7

Baes M. et al., 2014, MNRAS, 444, L90

Barazza F. D., Binggeli B., 2002, A\&A, 394, L15

Battinelli P., Demers S., 2004, A\&A, 417, 479

Bekki K., Couch W. J., Drinkwater M. J., 2001, ApJ, 552, L105

Bianchi S., Schneider R., 2007, MNRAS, 378, 973

Bica E., Alloin D., Schmidt A. A., 1990, A\&A, 228, 23

Bolatto A. D., Leroy A. K., Rosolowsky E., Walter F., Blitz L., 2008, ApJ, 686, 948

Boselli A., Gavazzi G., Lequeux J., Pierini D., 2002, A\&A, 385, 454

Boselli A., Boissier S., Cortese L., Gavazzi G., 2008, ApJ, 674, 742

Brandt W. N., Ward M. J., Fabian A. C., Hodge P. W., 1997, MNRAS, 291, 709

Brauher J. R., Dale D. A., Helou G., 2008, ApJS, 178, 280-301

Braun R., Thilker D. A., Walterbos R. A. M., Corbelli E., 2009, ApJ, 695, 937

Bresolin F., Stasińska G., Vílchez J. M., Simon J. D., Rosolowsky E., 2010, MNRAS, 404, 1679

Burton M. G., Hollenbach D. J., Tielens A. G. G., 1992, ApJ, 399, 563

Cigan P. et al., 2016, AJ, 151, 14

Cormier D. et al., 2010, A\&A, 518, L57

Cormier D. et al., 2014, A\&A, 564, A121

Crnojević D. et al., 2014, MNRAS, 445, 3862

Dame T. M., Hartmann D., Thaddeus P., 2001, ApJ, 547, 792

Davidge T. J., 2005, AJ, 130, 2087

De Looze I. et al., 2012, MNRAS, 423, 2359

De Looze I. et al., 2016, MNRAS, 459, 3900

De Rijcke S., Prugniel P., Simien F., Dejonghe H., 2006, MNRAS, 369, 1321

De Young D. S., Heckman T. M., 1994, ApJ, 431, 598

Draine B. T., Li A., 2007, ApJ, 657, 810

Engelbracht C. W., Rieke G. H., Gordon K. D., Smith J.-D. T., Werner M. W., Moustakas J., Willmer C. N. A., Vanzi L., 2008, ApJ, 678, 804

Evslin J., 2014, MNRAS, 440, 1225

Faber S. M., Gallagher J. S., 1976, ApJ, 204, 365

Ferrara A., Tolstoy E., 2000, MNRAS, 313, 291

Ferrara A., Viti S., Ceccarelli C., 2016, MNRAS, 463, L112

Fich M., Hodge P., 1991, ApJ, 374, L17

Finkelman I., Brosch N., Funes J. G., Kniazev A. Y., Väisänen P., 2010, MNRAS, 407, 2475

Frerking M. A., Keene J., Blake G. A., Phillips T. G., 1989, ApJ, 344, 311

Galametz M., Madden S. C., Galliano F., Hony S., Bendo G. J., Sauvage M., 2011, A\&A, 532, A56

Galametz M. et al., 2013, MNRAS 431, 1956

Galliano F., Dwek E., Chanial P., 2008, ApJ, 672, 214

Geha M., van der Marel R. P., Guhathakurta P., Gilbert K. M., Kalirai J., Kirby E. N., 2010, ApJ, 711, 361

Geha M., Weisz D., Grocholski A., Dolphin A., van der Marel R. P., Guhathakurta P., 2015, ApJ, 811, 114

Glover S. C. O., Clark P. C., 2016, MNRAS, 456, 3596

Goldsmith P. F., Langer W. D., Pineda J. L., Velusamy T., 2012, ApJS, 203, 13

Gonçalves D. R., Magrini L., Leisy P., Corradi R. L. M., 2007, MNRAS, 375,715

Gonçalves D. R., Magrini L., Martins L. P., Teodorescu A. M., Quireza C., 2012, MNRAS, 419, 854

Gonçalves D. R., Magrini L., Teodorescu A. M., Carneiro C. M., 2014, MNRAS, 444, 1705

Han M. et al., 1997, AJ, 113, 1001
Hodge P. W., 1973, ApJ, 182, 671

Howley K. M., Geha M., Guhathakurta P., Montgomery R. M., Laughlin G., Johnston K. V., 2008, ApJ, 683, 722

Hughes T. M. et al., 2015, A\&A, 575, A17

Hunt L., Bianchi S., Maiolino R., 2005, A\&A, 434, 849

Ikeda M., Oka T., Tatematsu K., Sekimoto Y., Yamamoto S., 2002, ApJS, 139,467

Israel F. P., Baas F., 2003, A\&A, 404, 495

James A., Dunne L., Eales S., Edmunds M. G., 2002, MNRAS, 335, 753

Kaufman M. J., Wolfire M. G., Hollenbach D. J., Luhman M. L., 1999, ApJ, 527,795

Kazantzidis S., Łokas E. L., Callegari S., Mayer L., Moustakas L. A., 2011, ApJ, 726, 98

Keene J., Blake G. A., Phillips T. G., Huggins P. J., Beichman C. A., 1985, ApJ, 299, 967

Kennicutt R. C., Jr, 1998, ApJ, 498, 541

Kerr A. R. et al., 2001, Sideband Calibration of Millimeter-Wave Receivers, ALMA Memo 357

Lanfranchi G. A., Matteucci F., 2004, MNRAS, 351, 1338

Lanfranchi G. A., Matteucci F., 2010, A\&A, 512, A85

Larson R. B., 1981, MNRAS, 194, 809

Lebouteiller V. et al., 2012, A\&A, 548, A91

Leroy A. K. et al., 2013, AJ, 146, 19

Lesaffre P., Pineau des Forêts G., Godard B., Guillard P., Boulanger F., Falgarone E., 2013, A\&A, 550, A106

Lisenfeld U., Ferrara A., 1998, ApJ, 496, 145

Lisker T., Weinmann S. M., Janz J., Meyer H. T., 2013, MNRAS, 432, 1162

McConnachie A. W., Irwin M. J., Ferguson A. M. N., Ibata R. A., Lewis G. F., Tanvir N., 2005, MNRAS, 356, 979

Madden S. C., 2000, New Astron. Rev., 44, 249

Madden S. C., Poglitsch A., Geis N., Stacey G. J., Townes C. H., 1997, ApJ, 483,200

Madden S. C., Cormier D., Remy-Ruyer A., 2016, preprint (arXiv:1603.04674)

Magrini L., Stanghellini L., Corbelli E., Galli D., Villaver E., 2010, A\&A, 512, AA63

Magrini L. et al., 2011, A\&A, 535, A13

Makiwa G., Naylor D. A., Ferlet M., Salji C., Swinyard B., Polehampton E., van der Wiel M. H. D., 2013, Appl. Opt., 52, 3864

Malhotra S. et al., 2001, ApJ, 561, 766

Marleau F. R., Noriega-Crespo A., Misselt K. A., 2010, ApJ, 713, 992

Martínez-Delgado D., Aparicio A., Gallart C., 1999, AJ, 118, 2229

Martins L. P., Lanfranchi G., Gonçalves D. R., Magrini L., Teodorescu A. M., Quireza C., 2012, MNRAS, 419, 3159

Mayer L., Governato F., Colpi M., Moore B., Quinn T., Wadsley J., Stadel J., Lake G., 2001, ApJ, 559, 754

Monaco L., Saviane I., Perina S., Bellazzini M., Buzzoni A., Federici L., Fusi Pecci F., Galleti S., 2009, A\&A, 502, L9

Nakajima T. et al., 2008, PASJ, 60, 435

Negishi T., Onaka T., Chan K.-W., Roellig T. L., 2001, A\&A, 375, 566

Oberst T. E. et al., 2006, ApJ, 652, L125

Papadopoulos P. P., Thi W.-F., Viti S., 2004, MNRAS, 351, 147

Parkin T. J. et al., 2013, ApJ, 776, 65

Parkin T. J. et al., 2014, ApJ, 787, 16

Paudel S., Ree C. H., 2014, ApJ, 796, L14

Pilbratt G. L. et al., 2010, A\&A, 518, L1

Planck Collaboration XIII, 2016, A\&A, 594, A13

Poglitsch A., Krabbe A., Madden S. C., Nikola T., Geis N., Johansson L. E. B., Stacey G. J., Sternberg A., 1995, ApJ, 454, 293

Poglitsch A. et al., 2010, A\&A, 518, L2

Pound M. W., Wolfire M. G., 2008, in Argyle R. W., Bunclark P. S., Lewis J. R., eds, ASP Conf. Ser. Vol. 394, Astronomical Data Analysis Software and Systems XVII. Astron. Soc. Pac., San Francisco, p. 654

Raiteri C. M., Villata M., Navarro J. F., 1996, A\&A, 315, 105

Rémy-Ruyer A. et al., 2014, A\&A, 563, A31

Richer M. G., McCall M. L., 2008, ApJ, 684, 1190

Roberts M. S., Hogg D. E., Bregman J. N., Forman W. R., Jones C., 1991, ApJS, 75, 751 
Roussel H. et al., 2007, ApJ, 669, 959

Ryś A., Falcón-Barroso J., van de Ven G., 2013, MNRAS, 428, 2980

Sage L. J., Welch G. A., Mitchell G. F., 1998, ApJ, 507, 726

Saintonge A. et al., 2011, MNRAS, 415, 61

Salpeter E. E., 1955, ApJ, 121, 161

Sawada T. et al., 2008, PASJ, 60, 445

Sawala T., Scannapieco C., White S., 2012, MNRAS, 420, 1714

Schirm M. R. P. et al., 2014, ApJ, 781, 101

Smith J. D. T. et al., 2016, ApJ, preprint (arXiv:1611.01521)

Sofia U. J., Cardelli J. A., Guerin K. P., Meyer D. M., 1997, ApJ, 482, L105

Stacey G. J., Geis N., Genzel R., Lugten J. B., Poglitsch A., Sternberg A., Townes C. H., 1991, ApJ, 373, 423

Stasińska G., Peña M., Bresolin F., Tsamis Y. G., 2013, A\&A, 552, AA12

Strong A. W., Mattox J. R., 1996, A\&A, 308, L21

Teyssier M., Johnston K. V., Kuhlen M., 2012, MNRAS, 426, 1808

Tolstoy E., Hill V., Tosi M., 2009, ARA\&A, 47, 371

Valluri M., Jog C. J., 1990, ApJ, 357, 367

van den Bergh S., 1998, AJ, 116, 1688 van den Hoek L. B., Groenewegen M. A. T., 1997, A\&AS, 123,

Walter F., Weiß A., Downes D., Decarli R., Henkel C., 2011, ApJ, 730, 18

Watkins L. L., Evans N. W., van de Ven G., 2013, MNRAS, 430, 971

Weiß A., Downes D., Henkel C., Walter F., 2005, A\&A, 429, L25

Welch G. A., Mitchell G. F., Yi S., 1996, ApJ, 470, 781

Welch G. A., Sage L. J., Mitchell G. F., 1998, ApJ, 499, 209

Wilson C. D. et al., 2013, ApJ, 776, L30

Wolfire M. G., Hollenbach D., McKee C. F., 2010, ApJ, 716, 1191

Woosley S. E., Weaver T. A., 1995, ApJS, 101, 181

Young L. M., 2001, AJ, 122, 1747

Young L. M., Lo K. Y., 1996, ApJ, 464, L59

Young L. M., Lo K. Y., 1997, ApJ, 476, 127

Zhukovska S., 2014, A\&A, 562, A76

Zhukovska S., Gail H.-P., Trieloff M., 2008, A\&A, 479, 453

This paper has been typeset from a $\mathrm{TE}_{\mathrm{E}} \mathrm{X} / \mathrm{L} \mathrm{T} \mathrm{E}$ file prepared by the author. 